\title{
Calcareous nannofossil bioevents at the Palaeocene/Eocene boundary in the Kharga Oasis, Western Desert of Egypt
}

\author{
Mahmoud Faris ${ }^{1}$, Manal Shabaan ${ }^{2}$ and Fatma Shaker ${ }^{3}$ \\ 1 Tanta University, Faculty of Science, Department of Geology, Tanta, Egypt; (corresponding author: mhmfaris@yahoo.com) \\ ${ }^{2}$ Kafr El Sheikh University, Faculty of Science, Department of Geology, Kafr El Sheikh, Egypt; (manalshabaan2010@yahoo.com) \\ ${ }^{3}$ Benha University, Faculty of Science, Department of Geology, Benha, Egypt; (fatma_elkammar@hotmail.com, fatma.eldesouky@fsc.bu.edu.eg)
}

doi: $10.4154 / g c .2017 .13$

Article history:

Manuscript received April 06, 2017

Revised manuscript accepted September 19, 2017 Available online October 31, 2017

Keywords: Calcareous nannofossils, Palaeocene/ Eocene boundary, Kharga Oasis, Western Desert, Egypt

\begin{abstract}
Two upper Palaeocene - lower Eocene stratigraphic sequences at the Kharga Oasis (Umm El Ghanayim and Naqb Assiut sections) were studied biostratigraphically on the basis of their calcareous nannofossil content. The investigated interval includes the upper part of the Tarawan Formation, the Tarawan Chalk, and the Esna formations. A total number of sixty-seven different taxa have been identified. The lowest occurrence (LO) of Discoaster araneus was used to place the base of the NP9b Subzone (base of Eocene) at the Gabal Umm El Ghanayim section. The lowest occurrences (LOs) of Rhomboaster bitrifida, Discoaster araneus and D. anartios are used to define the NP9a/NP9b subzonal boundary at the Gabal Naqb Assiut section. In this section, the P/E boundary is marked by a minor lithologic hiatus as indicated by the absence of the basal part of the Dababiya Member.

At the studied two sections, a major turnover in calcareous nannofossil assemblages across the $\mathrm{P} / \mathrm{E}$ transition was documented. The abundance of warm water Ericsonia subpertusa, Fasculithus spp., Coccolithus eopelagicus, Discoaster spp., Rhomboaster bitrifida and Tribrachiatus bramlettei characterize the Palaeocene-Eocene transition and suggest global warming and the Palaeocene - Eocene Thermal Maximum (PETM).
\end{abstract}

\section{INTRODUCTION}

The late Palaeocene to early Eocene interval is considered to be one of the warmest time periods (THOMAS, 1998) known as the Palaeocene - Eocene Thermal Maximum (PETM) (KENNETT \& STOTT, 1991) during which numerous events occurred. The remarkable climate change during that time can be explained by the release of a massive amount of methane from gas hydrates (DICKENS et al., 1995; KATZ et al., 1999).

Major changes in the terrestrial and marine biota occurred at the PETM. This interval is marked by the mass extinction of benthic foraminifera, with a global decrease in their diversity, which ranges from 30 to 50\% (KENNETT \& STOTT, 1991), being known as the Benthic Foraminiferal Extinction Event (BFEE) (THOMAS \& SHACKLETON, 1996). The time interval is characterized by the presence of short-ranging taxa such as „malformed discoasters" and ,rhomboasters". The extreme environmental changes at the PETM caused those malformations of coccoliths (JIANG \& WISE, 2006). The global Palaeocene/Eocene boundary was defined at the Dababiya Quarry section, south of Luxor, in the Upper Nile Valley (DUPUIS et al., 2003).

The aims of the present study are to identify the calcareous nannofossil assemblages of the PETM interval, to assign the studied sections to several nannofossil biozones and to provide some light on the palaeoecology for the late Palaeocene-early Eocene interval based on the presence of the most abundant calcareous nannofossil taxa.

\section{GEOLOGIC SETTING AND LITHOSTRATIGRAPHY}

The Kharga Oasis is one of the depressions of the Western Desert, lying between latitudes $24^{\circ}$ and $26^{\circ}$ north, about $200 \mathrm{~km}$ to the west of the Nile Valley. The two sections at Kharga Oasis are the Naqb Assiut and Gabal Umm El Ghanayim sections were investigated (Fig. 1).

The upper Palaeocene to lower Eocene sedimentary succession in the Kharga Oasis includes from the base to the top: the upper part of the Dakhla Shale, the Tarawan Chalk, the Esna Shale and the Thebes formations. The lithostratigraphic correlation for the $\mathrm{P} / \mathrm{E}$ rock units in different sections from the western and eastern Desert and the present study, are illustrated in Table 1.

In the following paragraphs a brief description of the measured and sampled formations is given.

\subsection{Tarawan Formation}

The Tarawan Formation was first termed by AWAD \& GHOBRIAL (1965) to describe the limestone; chalky limestone and the marly limestone succession which overlie the Dakhla Shale and underlie the Esna Formation. This unit is about $11 \mathrm{~m}$ thick, and is represented by samples 1 to 8 in the Gabal Umm El Ghanayim section.

\subsection{Esna Formation}

The term Esna Shale was first introduced by BEADNELL (1905), to describe the shale succession that overlies the Tarawan Formation and underlies the Thebes Formation at Gabal Oweina, EsnaIdfu area, Upper Nile Valley. ABDEL-RAZIK (1972) separated this formation into two members, the El Hanadi Member at the base and the El Shaghab Member at the top. DUPUIS et al. (2003) classified the Esna Formation from the base to the top, into three units (Esna Unit 1, Esna Unit 2 and Esna Unit 3) (Table 1). 


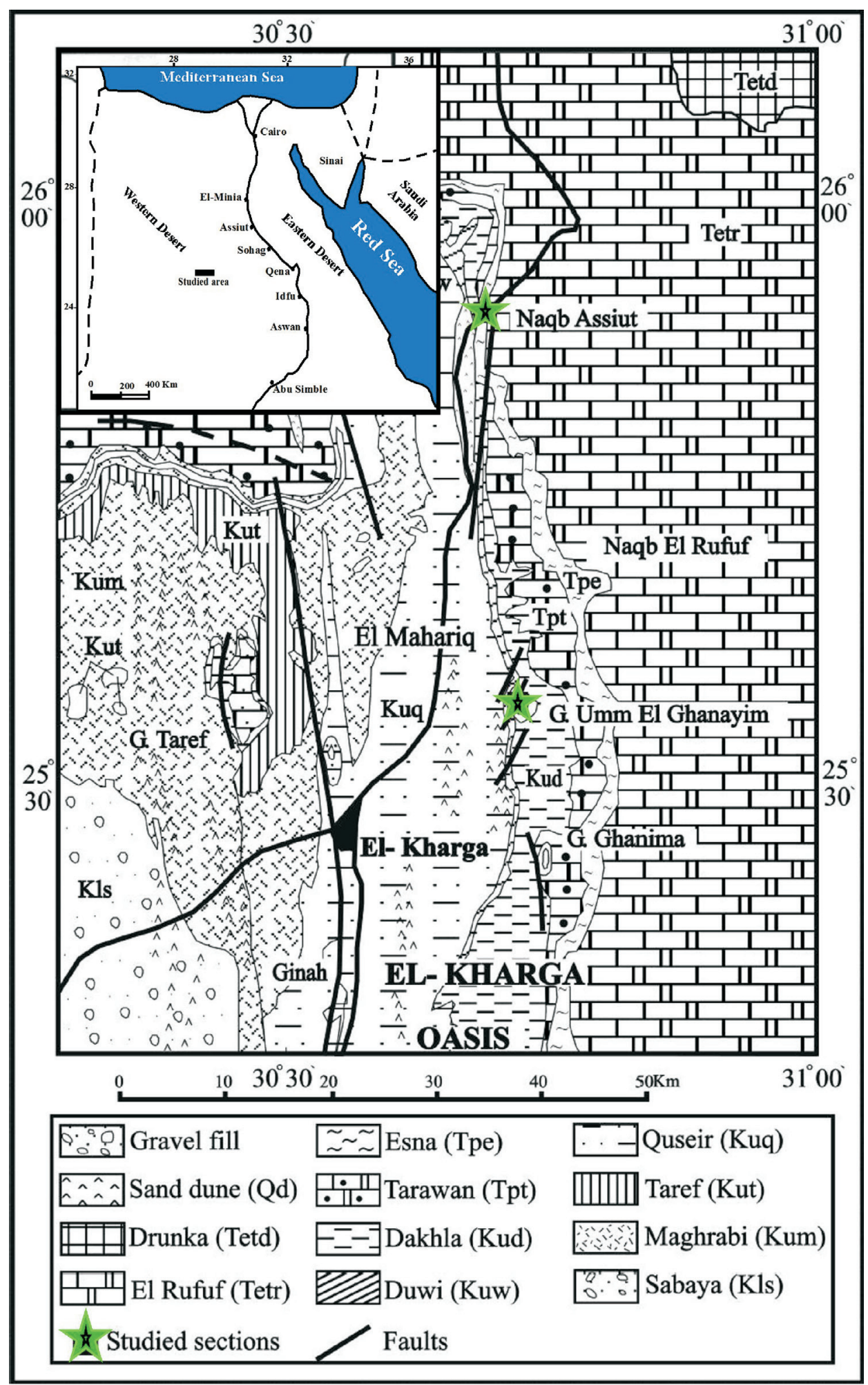

Figure 1. Geological map of the Kharga area (Western Desert, Egypt) with the location of the studied sections (modified after SAID, 1990).

AUBRY et al. (2007) subdivided the Esna Formation into four members, from the base to the top as follows, the El Hanadi, El Dababiya Quarry, El Mahmiya and Abu Had members.

In the present study, the Esna Formation was sampled and described. The total thickness is about $17.5 \mathrm{~m}$ at the Gabal Umm El Ghanayim section and about $10 \mathrm{~m}$ at the Naqb Assiut section. This rock unit represents the El-Hanadi, El Dababiya Quarry and the lower part of El-Mahmiya Member (Figs. 2 and 3). These members will be discussed briefly below.

\subsubsection{El Hanadi Member}

The El Hanadi Member was originally introduced by ABDELRAZIK (1972) to describe the lower part of the Esna Formation ( $5 \mathrm{~m}$ of shale succession) which overlies the Tarawan Formation and underlies the El Shaghab Member at Gabal El Shaghab, east of El Hanadi Village, near Esna City. According to AUBRY et al. (2007), the El Hanadi Member equates to the Esna Unit 1 of DUPUIS et al. (2003). At the Gabal Umm El Ghanayim section, the El Hanadi Member is composed of a calcareous shale about $1 \mathrm{~m}$ 
thick at the base (sample 9 to 11), and another shale of about $8 \mathrm{~m}$ thick (samples 12 to 26) at its top (Fig. 2). At the Naqb Assiut section, the lower part of the El Hanadi Member is composed of about $6 \mathrm{~m}$ of shale deposits (samples 1 to 17) and varies gradually into massive marls of about $0.95 \mathrm{~m}$ thick (samples 18 and 19) in its upper part.

\subsubsection{Dababiya Quarry Member}

At the type locality, the El Dababiya Quarry Member consists of five characteristic beds (1-5) of about $3.68 \mathrm{~m}$ thickness: Bed no. 1 is an organic rich clay layer ( $\sim .63 \mathrm{~m}$ thick); bed no. 2 is a phosphate brown shale ( $\sim 0.50 \mathrm{~m}$ thick); and bed no. 3 is a creamy phosphatic shale ( $\sim 0.84 \mathrm{~m}$ thick); bed no. 4 is a grey calcareous shale ( $\sim 07.1 \mathrm{~m}$ thick) and bed no. 5 is a calcarenitic marly limestone ( $\sim 1.00 \mathrm{~m}$ thick) (AUBRY et al., 2007).

In the current study, the Dababiya Quarry Member is about $1.2 \mathrm{~m}$ thick at Gabal Umm El Ghanayim and $0.9 \mathrm{~m}$ thick at the Naqb Assiut section. This Member consists of three beds as described from the base to the top: Bed 1 is an organic rich clay layer, which contains scattered coprolites at Gabal Umm El Ghanayim (sample 27, about $15 \mathrm{~cm}$ ), but it is absent at the Naqb Assiut section; Bed 2 is brown-coloured fish debris and coprolite-rich laminated shale at Gabal Umm El Ghanayim (samples 28 to 35, about $90 \mathrm{~cm}$ ). At the Naqb Assiut section it consists of massive phosphatic marls rich with collophane grains at the base, and $70 \mathrm{~cm}$ of marly limestone at the top (samples 20 and 21, about $20 \mathrm{~cm}$ ). Bed 3 consists of marls at Gabal Umm El-Ghanayim (sample 36, about $15 \mathrm{~cm}$ ), while at the Naqb Assiut section (Fig. 3) it is mainly composed of chalky limestone (samples 22 to 25 , about $70 \mathrm{~cm}$ ).

\subsubsection{El Mahmiya Member}

This Member was defined by AUBRY et al. (2007), to cover the Esna 2 Unit of DUPUIS et al. (2003) at the Village of El Dababiya
(35 km south of Luxor). At the Gabal Umm El Ghanayim section, the El Mahmiya Member is essentially composed of about $6 \mathrm{~m}$ of shale (samples 37 to 48), while at the Naqb Assiut section, the El Mahmiya Member consists mainly of $2.25 \mathrm{~m}$ of calcareous shale (samples 26 to 30 ).

\section{MATERIAL AND METHODS}

A total number of 78 samples were collected for calcareous nannofossils investigations, from which 48 belong to the Gabal Umm El Ghanayim section and 30 to Naqb Assiut. The samples have been closely collected from the upper Palaeocene-lower Eocene interval in the study sections and reach about $5 \mathrm{~cm}$ near the P/E boundary. The calcareous nannofossil assemblages were observed using a polarized light microscope at a magnification of $1250 \mathrm{X}$ and their identification was based on the standard taxonomy of PERCH-NIELSEN (1985).

Calcareous nannofossil abundances were determined by counting 200-300 specimens/slide (BACKMAN \& SHAKLETON, 1983). Additionally, one random traverse of the slide was scanned for rarer species. The nannofossil relative abundance was considered as follows: $\mathrm{A}=$ abundant $(>10$ specimens/field of view), $\mathrm{C}=$ common (1-10 specimens/field of view), $\mathrm{F}=$ few $(1$ specimen/1-10 fields of view), $\mathrm{R}=$ rare $(1$ specimen $/>10$ fields of view) $\mathrm{VR}=$ very rare (1 specimen/more than 100 field of view). The species richness is given as the total number of species recorded in each sample.

Nannofossil preservation was classified as: $\mathrm{G}=\operatorname{good}$ (little or no evidence of dissolution and/or overgrowth; specimens can be easily identified to species level) and $\mathrm{M}=$ moderate (specimens exhibit some etching and/or overgrowth; most specimens are identifiable to the species level).

Table 1. Lithostratigraphic correlation for the P/E transition interval, between different works from the western and eastern Desert and the present study.

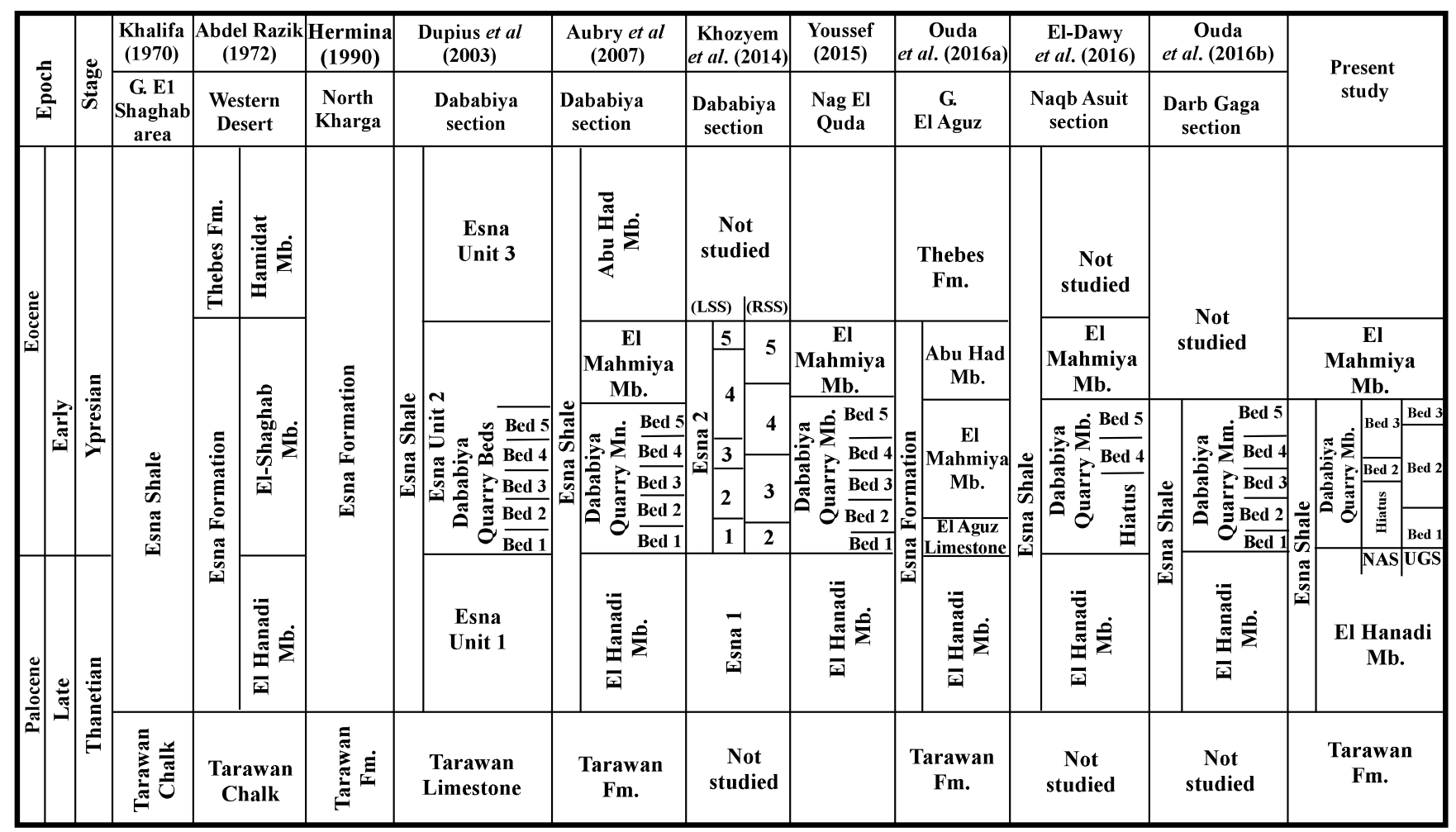



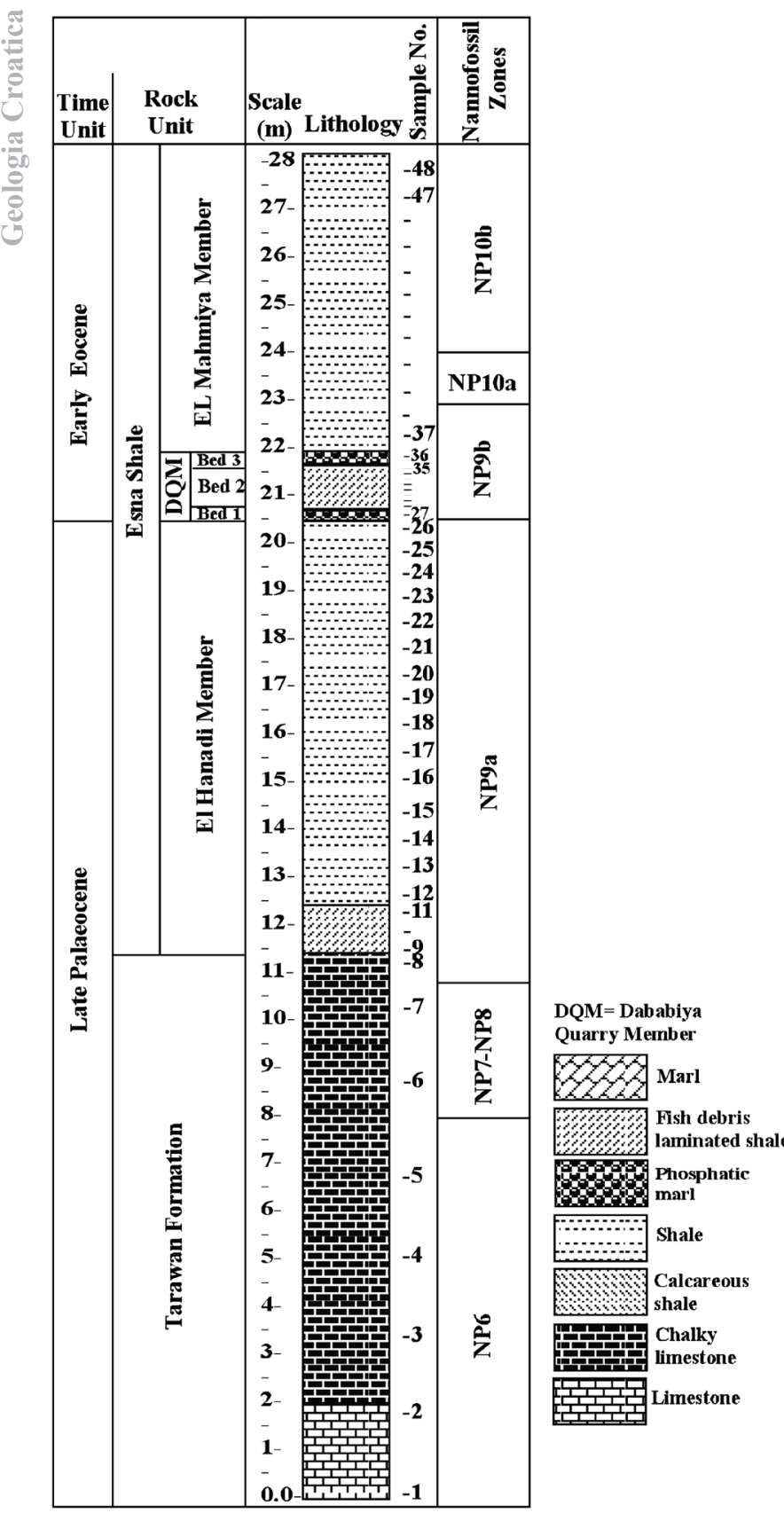

Figure 2. Lithostratigraphy and biostratigraphy of Umm El Ghanayim section (Kharga Oasis, Western Desert, Egypt), samples position and correlation to the standard zonation of MARTINI (1971).

Palaeotemperatures from calcareous nannofossil proxies were calculated using the Microsoft Excel program in accordance with the mentioned authors in the palaeoecologysection and our research data.

The biostratigraphic zonation proposed by MARTINI (1971) and OKADA \& BUKRY (1980) was adopted. In addition, other bioevents for lower latitudes were also used (AGNINI et al., 2014).

\section{RESULTS}

\subsection{Calcareous Nannofossils Biostratigraphy}

The present manuscript concerns the biostratigraphy of calcareous nannofossils from the upper part of the Tarawan Chalk and Esna formations (upper Palaeocene - lower Eocene interval) at the Umm El Ghanayim and Naqb Assiut sections.

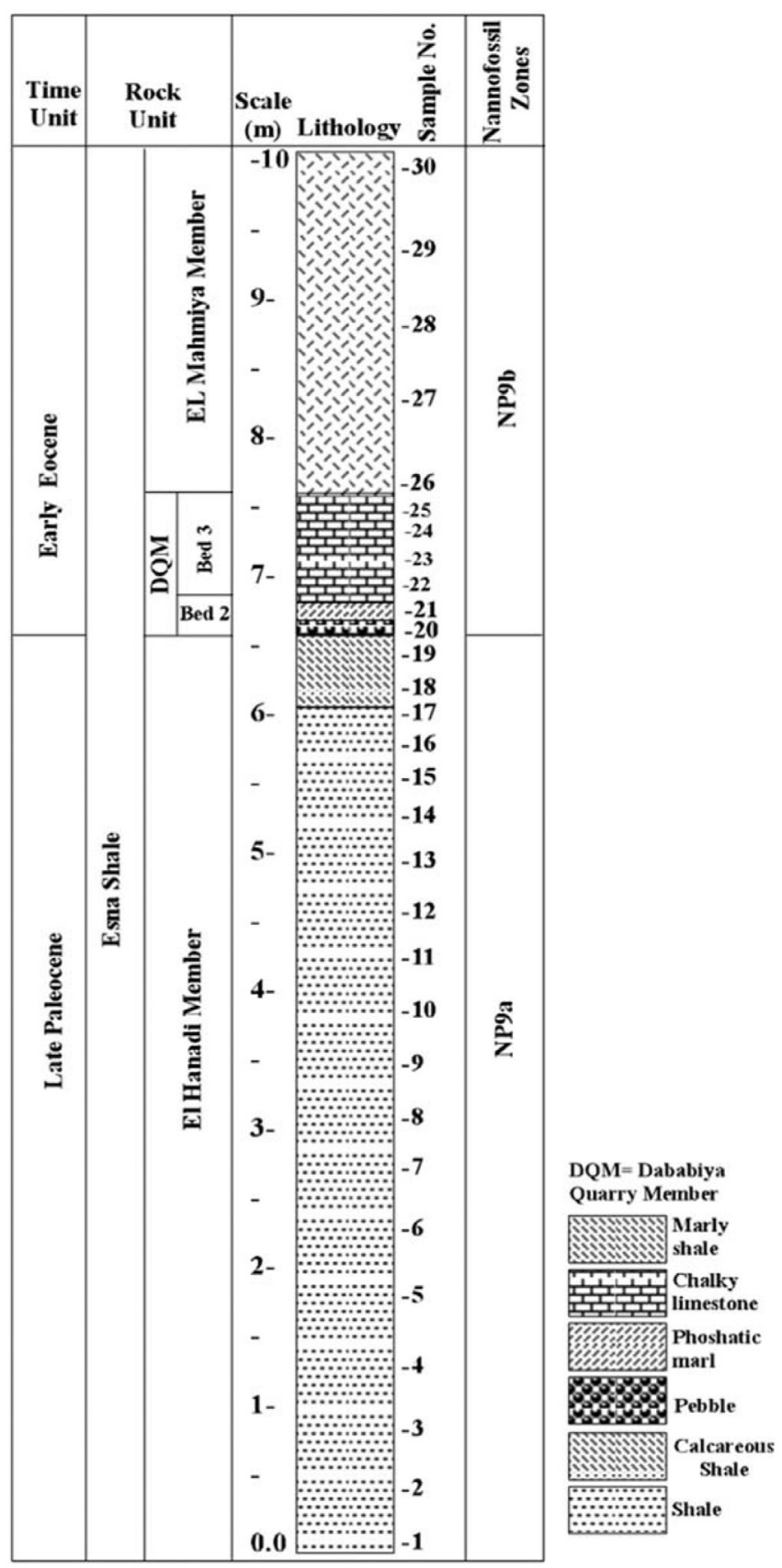

Figure 3. Lithostratigraphy and biostratigraphy of Naqb Assiut section (Kharga Oasis, Western Desert, Egypt), samples position and correlation to the standard zonation of MARTINI (1971).

The calcareous nannofossil assemblages are rich (72 species) and very diverse (22 genera) throughout the studied interval (late Palaeocene to early Eocene), while the preservation varies from moderate to good.

The stratigraphic distribution charts of calcareous nannofossil species are shown in Figs. 4 and 5. Some index calcareous nannofossil species are illustrated in Pls. 1 and 2.

Abbreviations used in the present study are: $\mathrm{NP}=$ Palaeogene nannofossil zone of MARTINI (1971), CP = Palaeogene nannofossil zone of OKADA \& BUKRY (1980), CNP (Palaeocene calcareous nannofossil zone) and CNE (Eocene calcareous nannofossil zone) of AGNINI et al. (2014). Their correlation is shown in Table 2 with four nannofossil zones identified in the present study, covering the time interval from the late Palaeocene to early 
Eocene and occupying the top of the Tarawan Chalk and the Esna Shale formations. These biozones are briefly discussed below.

\subsubsection{Heliolithus kleinpellii Zone (NP6)}

Definition: This zone comprises the interval from the LO of Heliolithus kleinpellii to the LO of Discoaster mohleri.

Authors: MOHLER \& HAY in HAY et al. (1967)

Age: Late Palaeocene

Correlation: H. kleinpellii Zone (CP5) of OKADA \& BUKRY (1980). It is equivalent to the upper part of the $H$. cantabriae BZ (CNP8) of AGNINI et al. (2014) (Table 2).

Occurrence: This zone is represented by 1-5 samples, from the Gabal Umm El Ghanayim section. It is not recorded at some localities in Egypt (FARIS et al., 1999; FARIS \& ZAHRAN, 2002). The H. kleinpellii Zone is well represented in many other areas in Egypt (STROUGO \& FARIS, 1993; FARIS et al., 2000, 2005; AYYAD et al., 2003; FARIS \& ABU SHAMA, 2007a; FARIS \& FAROUK, 2015a).

Remarks on assemblages: Beside the marker species $\mathrm{He}$ liolithus kleinpellii (Fig. 4), this zone includes Coccolithus eopelagicus, Ericsonia subpertusa, E. universa, Toweius eminens and T. tovae.

\subsubsection{Discoaster mohleri- Heliolithus riedelii Zone (NP7-NP8)}

Definition: This zone is defined as the interval from the LO of Discoaster mohleri to the LO of Discoaster multiradiatus.

Author: HAY (1964); emended by ROMEIN (1979).

Age: Late Palaeocene

Correlation: It is equivalent to the $D$. mohleri $-D$. nobilis (CP6-CP7) Zones of OKADA \& BUKRY (1980), to the Discoaster mohleri (CNP9) and Discoaster backmanii (CNP10) Zones of AGNINI et al. (2014) (Table 2).
Occurrence: At Gabal Umm El Ghanayim, this zone is restricted to within the top part of the Tarawan Formation (samples 6 and 7). Discoaster mohleri/Heliolithus riedelii Zone was recorded in different areas in Egypt (FARIS 1988a, b; STROUGO \& FARIS, 1993; FARIS et al., 1999; AYYAD et al., 2003; GHANDOUR et al,. 2004; FARIS \& ABU SHAMA, 2007a; FARIS \& SALEM, 2007b).

Remarks on assemblages: The base of this zone is defined by the LO of $D$. mohleri whilst its top is defined by the LO of $D$. multiradiatus (ABU SHAMA et al., 2007; FARIS \& ABU SHAMA, 2007a). The calcareous nannofossil assemblages are similar to those of the underlying NP6 Zone, with the additional presence of marker species D. mohleri (Fig. 4).

\subsubsection{Discoaster multiradiatus Zone (NP9)}

Definition: This zone defines the interval from the LO of Discoaster multiradiatus to the LO of Tribrachiatus bramlettei.

Authors: BRAMLETTE \& SULLIVAN (1961); amended by MARTINI (1971)

Age: Late Palaeocene

Correlation: Chiasmolithus bidens Subzone (CP8a) of OKADA \& BUKRY (1980). It is equivalent to the Discoaster multiradiatus/Fasciculithus richardii gr. CRZ (CNP11) of AGNINI et al. (2014) (Table 2).

Occurrence: The NP9 Zone covers the topmost part of the Tarawan Formation (sample 8), as well as most of the Esna Formation (samples 9 to 38) at the Gabal Umm El Ghanayim section, and occupies the whole Esna Formation at the Gabal Naqb Assiut section (samples 1 to 30 ).

Remarks on assemblages: The calcareous nannofossil assemblages are highly diverse and abundant in the NP9 Zone and this agrees well with the statement of PERCH-NIELSEN (1985) that the Palaeocene diversity reached a maximum value in the

Table 2. Late Palaeocene to Early Eocene biozones and bioevents, according to the standard biozonations of MARTINI, 1971 (NP), OKADA \& BUKRY, 1980 (CN), AGNINI et al., 2014 (CNP to CNE) and the zonal scheme of the present study.

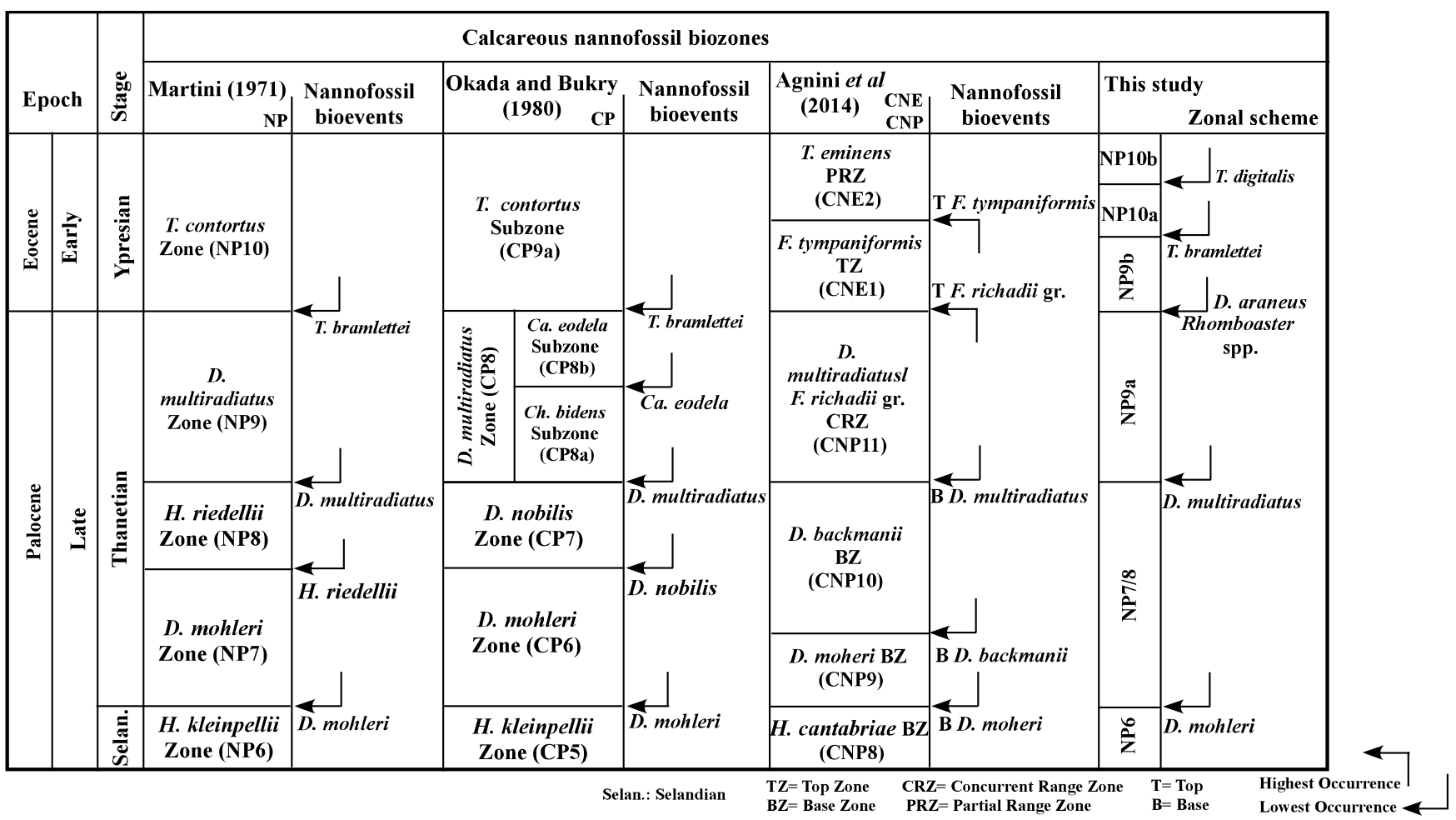


NP9 Zone. The identification of NP9 is mainly based on the presence of $D$. multiradiatus. The marker species $D$. multiradiatus is continuously present from sample 8 upwards in G. Umm El Ghanayim and appears in all samples in the Nabq Assiut section. The genus Fasciculithus suffered decreasing diversity at the onset of the $\mathrm{P} / \mathrm{E}$ boundary, and disappeared completely shortly above the base of the NP10 Zone.

AUBRY (1999) divided the NP9 Zone into two subzones (NP9a and NP9b) based on the lowest appearance of Rhomboaster spp. and/or Discoaster araneus.

\begin{tabular}{|c|c|c|c|c|c|c|c|c|c|c|c|c|c|c|c|c|c|c|c|c|c|c|c|c|}
\hline & & & & & & & & & Late & Paleod & ocene & & & & & & & & & & & & & \\
\hline & & & & & & & & & & & & & & & & & & & na & Sha & & & & \\
\hline & & & Tara & awa & & & & & & & & & L H & Jamdi & & & & & & & & & abal & biya \\
\hline- & $N$ & $\omega$ & $\rightarrow$ & ur & a) & +0 & $\infty \quad 0$ & $\overline{0}$ & $=\pi$ & $\bar{\omega} \mid \mp$ & $\overline{\boldsymbol{v}}$ & $\bar{a}$ & $\Xi$ & $\bar{\infty} \mid \overline{0}$ & $\stackrel{0}{6}$ & $\underline{\simeq}$ & $\mathbf{N}$ & $\tilde{\omega}$ & $\stackrel{N}{ \pm}$ & Nut & $\tilde{\sigma}$ & ज. & $\approx$ & • \\
\hline x] & $\mathbf{x}$ & $\Rightarrow$ & $\mathrm{x}$ & $\mathbf{x}$ & $\boldsymbol{\nabla}$ & $0:$ & $>>$ & $>$ & $>>$ & 00 & 0 & $>$ & $>$ & $>0$ & $a>$ & $>$ & $>$ & $>$ & O & 0 & ๑) & x $=$ & 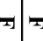 & $\Rightarrow x$ \\
\hline 3 & 3 & 3 & 3 & 3 & $\leq 6$ & $\Omega$ & $\therefore \Omega$ & $a$ & $\Omega 0$ & $a \Omega$ & $a$ & $\Omega$ & a & $a \Omega$ & $\Omega \Omega$ & 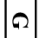 & $a$ & a & a & 3 & 3 & $3=$ & 3 & 3 \\
\hline & & NP6 & & & 等 & & & & & & & & JP9a & & & & & & & & & & & \\
\hline $\mathbf{F}$ & $\mid \mathbf{F}$ & C & \begin{tabular}{|l|}
$\mathbf{F}$ \\
\end{tabular} & \begin{tabular}{l|l|}
$\mathbf{F}$ & \\
\end{tabular} & \begin{tabular}{l|l}
$\mathbf{F}$ & \\
\end{tabular} & $\mathbf{F}$ & \begin{tabular}{|l|l|}
$\mathbf{F}$ & $\mathbf{R}$ \\
\end{tabular} & \begin{tabular}{|l|}
$\mathbf{R}$ \\
\end{tabular} & \begin{tabular}{l|l}
$F$ & $F$ \\
\end{tabular} & \begin{tabular}{|l|l|}
$\mathbf{R}$ & $\mathbf{F}$ \\
\end{tabular} & \begin{tabular}{|l|}
$\mid \mathbf{C}$ \\
\end{tabular} & \begin{tabular}{|l|}
$\mathrm{F}$ \\
\end{tabular} & \begin{tabular}{|l|}
$\mathbf{F}$ \\
\end{tabular} & \begin{tabular}{|l|}
$\mathbf{F}$ \\
\end{tabular} & \begin{tabular}{|l|l|}
$\mathbf{F}$ & $\mathbf{F}$ \\
\end{tabular} & \begin{tabular}{|l|}
$F$ \\
\end{tabular} & $\mathbf{F}$ & \begin{tabular}{|l|}
$\mathbf{F}$ \\
\end{tabular} & $\mathbf{F}$ & \begin{tabular}{|l|}
$\mathbf{F}$ \\
\end{tabular} & \begin{tabular}{l|l}
$\mathbf{C}$ \\
\end{tabular} & 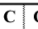 & \begin{tabular}{l|l}
$\mathbf{C}$ & $\mathbf{C}$ \\
\end{tabular} & $\mathbf{F}$ \\
\hline $\mathbf{R}$ & VR & & & $\mathbf{R}$ & $\begin{array}{lll} & 1 \\
\end{array}$ & $\mathbf{R}$ & VR & & $\mathbf{F}$ & $\mathbf{R}$ & & & VR & & \begin{tabular}{|l|} 
\\
\end{tabular} & & $\mathbf{R}$ & & & $\mathbf{F}$ & F & $\begin{array}{l} \\
F\end{array}$ & \begin{tabular}{|l|l}
$\mathbf{F}$ & $\mathbf{F}$ \\
\end{tabular} & \\
\hline $\mathbf{F}$ & $\mathbf{F}$ & F & $\mathbf{F}$ & $\mathbf{F}$ & \begin{tabular}{l|l} 
F &
\end{tabular} & $\mathbf{F}$ & \begin{tabular}{|l|l|}
$F$ & $F$ \\
\end{tabular} & F & \begin{tabular}{|l|l|}
$\mathbf{F}$ & $\mathbf{F}$
\end{tabular} & \begin{tabular}{l|l}
$\mathbf{F}$ & $\mathbf{F}$ \\
\end{tabular} & & $\mathrm{F}$ & $\mathbf{F}$ & F & \begin{tabular}{|l|l|}
$\mathbf{R}$ & $\mathbf{F}$ \\
\end{tabular} & F & $\mathbf{F}$ & $\mathbf{F}$ & $\mathbf{F}$ & $\mathbf{F}$ & $\mathrm{F}$ & $\mathrm{C}$ & \begin{tabular}{|l|l}
$\mathbf{F}$ & $\mathbf{F}$
\end{tabular} & C \\
\hline $\mathrm{F}$ & $\mathbf{F}$ & $\mathrm{F}$ & $\mathbf{F}$ & F & $\mathrm{F}$ & $\mathbf{F}$ & \begin{tabular}{l|l}
$F$ & $F$
\end{tabular} & $\mathbf{F}$ & \begin{tabular}{|l|l}
$F$ & $F$ \\
\end{tabular} & $\mathrm{~F}$ & $\mathbf{F}$ & $\mathrm{F}$ & $\mathbf{F}$ & F & \begin{tabular}{|l|l|}
$\mathbf{F}$ & $\mathrm{R}$ \\
\end{tabular} & F & $\mathbf{F}$ & F & $\mathrm{F}$ & F & $\mathrm{F}$ & & $\begin{array}{lll}\mathbf{F} & \mathbf{R} \\
\end{array}$ & F \\
\hline $\mathbf{R}$ & $\mathbf{R}$ & $F$ & VR & $\mathbf{R}$ & \begin{tabular}{|l|}
$\mathbf{R}$ \\
\end{tabular} & \begin{tabular}{l|l}
$\mathrm{R}$ & $\mathrm{V}$
\end{tabular} & \begin{tabular}{|l|l|} 
VR & |
\end{tabular} & \begin{tabular}{|l|}
$\mathbf{R}$ \\
\end{tabular} & \begin{tabular}{|l|l|}
$R$ & $V R$ \\
\end{tabular} & \begin{tabular}{|l|l|l} 
& $V R$ \\
\end{tabular} & \begin{tabular}{|l|l|} 
\\
\end{tabular} & & R & VR VF & \begin{tabular}{|l|l|}
$\mathrm{R}$ & $\mathbf{R}$ \\
\end{tabular} & $\mathbf{R}$ & VR & VR & $\mathbf{R}$ & & & & & \\
\hline $\mathbf{R}$ & $\mathbf{R}$ & R & R & \begin{tabular}{l|l}
$\mathbf{R}$ &
\end{tabular} & VR V & VR/V & VR VR & $\mathbf{R}$ & VR & VR VR & VR & $\mathbf{R}$ & $\mathbf{R}$ & & \begin{tabular}{|l|l|}
$\mathbf{R}$ & $\mathbf{R}$ \\
\end{tabular} & & & & $\mathbf{R}$ & & & & & \\
\hline VR & $\mathbf{R}$ & & $\mathbf{R}$ & $\mathbf{R}$ & & $\mathbf{R}$ & \begin{tabular}{|l|l|}
$\mathbf{R}$ & $\mathbf{R}$ \\
\end{tabular} & & VR & VR & VR & & $\mathbf{R}$ & & & & & & $\mathbf{R}$ & $\mathbf{R}$ & & & & \\
\hline$\overline{V R}$ & $2 \mathrm{VR}$ & & & \begin{tabular}{|l|}
$\mathbf{R}$ \\
\end{tabular} & & $\mathbf{R}$ & \begin{tabular}{|l|}
$\mathbf{R}$ \\
\end{tabular} & \begin{tabular}{|l|}
$\mathbf{R}$ \\
\end{tabular} & \begin{tabular}{|l|l|}
$\mathbf{R}$ & $\mathbf{F}$ \\
\end{tabular} & & & & & & $\mathbf{R}$ & & & & & & & & & \\
\hline $\mathbf{R}$ & & $\mathbf{R}$ & & \begin{tabular}{l|l} 
R &
\end{tabular} & VR & & & & & & & & & & & & $\mathbf{R}$ & VR & $\mathbf{R}$ & $\mathbf{R}$ & $\mathbf{R}$ & \begin{tabular}{l|l}
$R$ & 1 \\
\end{tabular} & \begin{tabular}{l|l}
$\mathbf{R}$ & $\mathbf{R}$ \\
\end{tabular} & \\
\hline $\mathbf{R}$ & $\mathbf{R}$ & $\mathbf{R}$ & $\mathbf{R}$ & $\mathbf{R}$ & & $\mathrm{VR}$ & & VR & VR VR & & & & & & & & & & & & & & & \\
\hline & & & & & VRI & & & & & & & & & & & & & & & & & & & \\
\hline & & & & & VR & & \begin{tabular}{|l|l|} 
& VR
\end{tabular} & VR & \begin{tabular}{|l|l|} 
VR & \\
\end{tabular} & & VR V & $\begin{array}{ll}\mathbf{V R} \\
\end{array}$ & $\mathbf{R}$ & $\mathbf{R}$ & \begin{tabular}{|l|l|}
$\mathbf{R}$ & $\mathbf{R}$ \\
\end{tabular} & $\mathbf{R}$ & $\mathbf{R}$ & $\mathbf{R}$ & $\mathbf{R}$ & & & & & \\
\hline & & & & & R & $\mathbf{F}$ & \begin{tabular}{|l|l|}
$F$ & $F$ \\
\end{tabular} & F & \begin{tabular}{l|l}
$F$ & $F$ \\
\end{tabular} & \begin{tabular}{|l|l|}
$\mathbf{F}$ & $\mathbf{R}$ \\
\end{tabular} & $\mathbf{F}$ & $\mathrm{F}$ & $\mathbf{F}$ & & \begin{tabular}{|l|l|}
$\mathbf{R}$ & $\mathbf{R}$ \\
\end{tabular} & R & $R$ & & $\mathrm{R}$ & & & & $\mathbf{R}$ & \\
\hline & & & & & VR I & $\mathbf{R}$ & & & & & & & & & & & & & & & & & & \\
\hline & & & & & $\begin{array}{lll}V_{1} & \end{array}$ & \begin{tabular}{l|l}
$\mathbf{R}$ & $\mathbf{1}$ \\
\end{tabular} & \begin{tabular}{|l|l|}
$\mathbf{R}$ & $\mathbf{R}$ \\
\end{tabular} & F & \begin{tabular}{|l|l|}
$F$ & $F$
\end{tabular} & \begin{tabular}{|l|l|}
$\mathbf{F}$ & $\mathbf{R}$ \\
\end{tabular} & $\mathbf{R}$ & \begin{tabular}{|l|}
$\mathbf{R}$ \\
\end{tabular} & $F$ & & \begin{tabular}{|l|l|}
$\mathbf{R}$ & $\mathbf{R}$ \\
\end{tabular} & $\mathbf{R}$ & $\mathbf{R}$ & $\mathbf{R}$ & $\mathbf{R}$ & $\mathbf{R}$ & & & & \\
\hline & & & & & \begin{tabular}{l|l}
$\mathbf{R}$ & $\mathbf{V}$
\end{tabular} & VR & VR VR & VR & VR & VR & & VR & & VR & & $\mathbf{R}$ & & VR & & $\mathbf{R}$ & & & & \\
\hline & & & & & & \begin{tabular}{l|l}
$\mathbf{R}$ & $\mathrm{V}$
\end{tabular} & VR VR & $\mathbf{R}$ & $\mathbf{R}$ & \begin{tabular}{|l|} 
VR \\
\end{tabular} & & & & & $R$ & & & & & & & & & \\
\hline & & & & & & VR & $\begin{array}{lll}\text { VR } & \text { | }\end{array}$ & $\mathbf{R}$ & $F$ & \begin{tabular}{|l|l|}
$F$ & $F$ \\
\end{tabular} & $\begin{array}{ll}\mathbf{R} & 1\end{array}$ & $\mathbf{R}$ & $\mathbf{R}$ & $\mathbf{R}$ & \begin{tabular}{|l|l}
$\mathbf{R}$ & $\mathbf{F}$ \\
\end{tabular} & $\mathrm{F}$ & $\mathbf{F}$ & $\mathbf{F}$ & $\mathbf{R}$ & $\mathbf{R}$ & F & $\mathbf{F}$ & \begin{tabular}{l|l}
$\mathbf{R}$ & $\mathbf{R}$ \\
\end{tabular} & \begin{tabular}{|l|l|}
$\mathbf{R}$ & $\mathrm{R}$ \\
\end{tabular} \\
\hline & & & & & & & \begin{tabular}{|l|l|}
$\mathbf{R}$ & $\mathbf{R}$ \\
\end{tabular} & \begin{tabular}{|l|}
$\mathbf{R}$ \\
\end{tabular} & VR & \begin{tabular}{|l|l}
$R$ \\
\end{tabular} & & & VR & $\mathbf{R}$ & $\mathbf{R}$ & $\mathbf{R}$ & VR & $\mathbf{R}$ & & $\mathbf{R}$ & & VR & \begin{tabular}{|l|l}
$\mathbf{R}$ \\
\end{tabular} & \\
\hline & & & & & & & \begin{tabular}{|l|l|}
$\mathbf{R}$ & $\mathbf{R}$ \\
\end{tabular} & F & \begin{tabular}{|l|l|}
$\mathbf{F}$ & $\mathbf{R}$ \\
\end{tabular} & \begin{tabular}{|l|l|}
$\mathbf{R}$ & $\mathbf{R}$ \\
\end{tabular} & & \begin{tabular}{|l|}
$\mathbf{R}$ \\
\end{tabular} & & $\mathbf{R}$ & $\mathbf{R}$ & & & $\mathbf{R}$ & $\mathbf{R}$ & & & & $\mathbf{R}$ & \\
\hline & & & & & & & VR & & & VR & & & & & & & & VR & $\mathrm{R}$ & & & $V R$ & & \\
\hline & & & & & & & VR $R$ & & $\mathbf{R}$ & & & VR & $\mathbf{R}$ & & & & & & & & & & & \\
\hline & & & & & & & \begin{tabular}{|l|l|}
$F$ & $F$ \\
\end{tabular} & $\mathbf{F}$ & \begin{tabular}{|l|l|}
$\mathbf{C}$ & $\mathbf{R}$
\end{tabular} & \begin{tabular}{|l|l}
$F$ & $F$ \\
\end{tabular} & $\mathbf{R}$ & F & C & $\mathbf{F}$ & $\mathbf{R}$ & $\mathbf{R}$ & $\mathbf{R}$ & F & $\mathbf{R}$ & $\mathbf{R}$ & \begin{tabular}{l|l} 
\\
\end{tabular} & \begin{tabular}{l|l} 
VR & .
\end{tabular} & \begin{tabular}{l|l}
$\mathbf{R}$ & $\mathbf{R}$ \\
\end{tabular} & \begin{tabular}{|l|l} 
\\
\end{tabular} \\
\hline & & & & & & & \begin{tabular}{|l|l|}
$\mathbf{R}$ & $\mathrm{VR}$
\end{tabular} & & & & VR & VR & R & $V \mathbf{R}$ & $\mathbf{V R}$ & & & $V R$ & & $\mathbf{R}$ & & & & \\
\hline & & & & & & & VR VR & VR & & VR & & & & & RVR & & & & & & & & & \\
\hline & & & & & & & & VR & & & & VR & & & & & & & & & & & & \\
\hline & & & & & & & VR & $\mathbf{R}$ & $\mathbf{R}$ & VR VR & $\mathbf{R}$ & VRI & VR & $V R$ & VR & & & VR & & \begin{tabular}{l|l}
$\mathbf{R}$ \\
\end{tabular} & & & \begin{tabular}{l|l}
$\mathbf{R}$ & $\mathbf{R}$
\end{tabular} & \begin{tabular}{|l|l|}
$\mathbf{R}$ & $\mathbf{R}$
\end{tabular} \\
\hline & & & & & & & $\mathbf{R}$ & \begin{tabular}{|l|}
$\mathbf{R}$ \\
\end{tabular} & $\mathbf{R}$ & VR & & & R & VRVE & & $\mathbf{R}$ & \begin{tabular}{|l|} 
R \\
\end{tabular} & & & & & & & \\
\hline & & & & & & & $\mathbf{R}$ & & \begin{tabular}{l|l}
$\mathbf{R}$ & $\mathbf{R}$ \\
\end{tabular} & & & $\mathbf{R}$ & & $\mathbf{R}$ & \begin{tabular}{|l|l}
$V R$ \\
\end{tabular} & & VR & & & & & & & \\
\hline & & & & & & & & $\mathbf{R}$ & VR & VR VR & & VR & $\mathbf{R}$ & $\mathbf{R}$ & \begin{tabular}{|l|l|}
$\mathbf{R}$ & $\mathbf{R}$ \\
\end{tabular} & $\mathbf{R}$ & & & $\mathbf{R}$ & & $\mathbf{R}$ & VR & & \\
\hline & & & & & & & & $\mathbf{V R}$ & \begin{tabular}{|l|l|}
$\mathbf{R}$ & $\mathrm{V}$
\end{tabular} & VR VR & & & & & RVR & & & & & & & & & \\
\hline & & & & & & & & & VR VR & VR VR & VR & \begin{tabular}{|l|l|} 
\\
\end{tabular} & R & VR R & \begin{tabular}{|l|l|}
$\mathbf{R}$ & $\mathbf{R}$ \\
\end{tabular} & R & & & & $\begin{array}{ll}\mathbf{R} \\
\end{array}$ & $\begin{array}{ll}\mathbf{R} \\
\end{array}$ & & & \\
\hline & & & & & & & & & \begin{tabular}{|l|l|}
$V R$ & $R$
\end{tabular} & VR & & VR & \begin{tabular}{|l|}
$R$ \\
\end{tabular} & VR VF & R VR & $\mathbf{R}$ & VR & & & & & & $\mathbf{R}$ & \\
\hline & & & & & & & & & VR & & & & & & & & & & & & & & & \\
\hline & & & & & & & & & & $\begin{array}{|ll|}\text { VR } & \text { R } \\
\end{array}$ & VR & & $\mathbf{R}$ & \begin{tabular}{|l|l}
$\mathbf{R}$ & $\mathbf{R}$ \\
\end{tabular} & $\mathbf{R}$ & $\mathbf{R}$ & & & & & & & & \\
\hline & & & & & & & & & & VR VR & VR & & & & & & & & & & & & & \\
\hline & & & & & & & & & & \begin{tabular}{|l|} 
VR \\
\end{tabular} & & & & & & & & & & & & & & \\
\hline & & & & & & & & & & VR R & $\mathbf{R}$ & & & & & & $\mathbf{R}$ & & & & & & & \\
\hline & & & & & & & & & & VR & & & & & & & & & & & & & & \\
\hline & & & & & & & & & & & VR & VR & VR & VR $R$ & \begin{tabular}{|l|l|}
$\mathbf{R}$ & $\mathbf{R}$ \\
\end{tabular} & $\mathbf{R}$ & VR & $\mathbf{R}$ & $\mathbf{R}$ & VR & & & $\mathbf{R}$ & $\mathbf{R}$ \\
\hline & & & & & & & & & & & & \begin{tabular}{|l|} 
\\
\end{tabular} & R & & & & & & & & & & & \\
\hline & & & & & & & & & & & & $\mathbf{R}$ & F & & \begin{tabular}{|l|l|}
$\mathbf{R}$ & $\mathbf{R}$ \\
\end{tabular} & & & VR & & & & & & \\
\hline & & & & & & & & & & & & $\begin{array}{ll}\text { VR } \\
\end{array}$ & & & \begin{tabular}{|l}
$\mathbf{V R}$ \\
\end{tabular} & & & & & VR & & & $\begin{array}{lll}V R & R\end{array}$ & \begin{tabular}{|l|l|} 
& $R$ \\
\end{tabular} \\
\hline & & & & & & & & & & & & VR & $\mathbf{R}$ & & & & $\mathbf{R}$ & & & & & & & \\
\hline & & & & & & & & & & & & \begin{tabular}{|l|}
$\mathbf{R}$ \\
\end{tabular} & \begin{tabular}{|l|}
$\mathbf{R}$ \\
\end{tabular} & \begin{tabular}{|l|l|} 
R \\
\end{tabular} & $R$ & & $\mathbf{R}$ & & $\mathbf{R}$ & & & & & \\
\hline & & & & & & & & & & & & VR & & $\mathbf{R}$ & \begin{tabular}{|l}
$V R$ \\
\end{tabular} & & & & $\mathbf{R}$ & & & & & \\
\hline & & & & & & & & & & & & \begin{tabular}{l|l}
$\mathbf{R}$ \\
\end{tabular} & & \begin{tabular}{l|l}
$\mathbf{R}$ & $\mathbf{R}$ \\
\end{tabular} & \begin{tabular}{|l|l|}
$\mathbf{R}$ & $\mathbf{R}$ \\
\end{tabular} & \begin{tabular}{|l|}
$\mathbf{R}$ \\
\end{tabular} & $\mathbf{R}$ & & $\mathbf{R}$ & VR & & & & \\
\hline & & & & & & & & & & & & & & VR & & & & & VR & & & & & \\
\hline & & & & & & & & & & & & & & $\mathbf{R}$ & \begin{tabular}{|l|l|}
$\mathbf{R}$ & $\mathbf{R}$ \\
\end{tabular} & & $\mathbf{R}$ & $\mathbf{R}$ & $\mathbf{R}$ & VR & & & & \\
\hline & & & & & & & & & & & & & & & & & & & & & & & $\begin{array}{lll}V R \\
\text { V }\end{array}$ & \\
\hline & & & & & & & & & & & & & & & & & & & & & & & VR VI & \\
\hline & & & & & & & & & & & & & & & & & & & & & & & & \\
\hline & & & & & & & & & & & & & & & & & & & & & & & & \\
\hline & & & & & & & & & & & & & & & & & & & & & & & & \\
\hline & & & & & & & & & & & & & & & & & & & & & & & & \\
\hline & & & & & & & & & & & & & & & & & & & & & & & & \\
\hline & $\pi$ & & & & & & & & & & & & & & & & & & & & & & & \\
\hline & $\frac{7}{3}$ & & & & & & & & & & & & & & & & & & & & & & & \\
\hline & & & & & & & & & & & & & & & & & & & & & & & & \\
\hline & & & & & & & & & & & & & & & & & & & & & & & & \\
\hline & & & & & & & & & & & & & & & & & & & & & & & & \\
\hline & & & & & & & & & & & & & & & & & & & & & & & & \\
\hline & & & & 38 & & & & & & & & & & & & & & & & & & & & \\
\hline & & & & 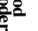 & & & & & & & & & & & & & & & & & & & & \\
\hline & & & & & & & & & & & & & & & & & & & & & & & & \\
\hline & & & & & & & & & & & & & & & & & & & & & & & & \\
\hline & & & & & & & & & & & & & & & & & & & & & & & & \\
\hline
\end{tabular}

Figure 4. Stratigraphic distribution of calcareous nannofossil species in Umm El Ghanayim section, Kharga Oasis, Western Desert, Egypt. 


\begin{tabular}{|c|c|c|c|c|c|c|c|c|c|c|c|c|c|c|c|c|c|c|c|c|c|c|c|c|c|c|c|}
\hline \multicolumn{17}{|c|}{ Late Paleocene } & \multicolumn{10}{|c|}{ Early Eocene } & Age \\
\hline \multicolumn{27}{|c|}{ Esna Shale } & \multirow{3}{*}{$\begin{array}{l}\text { Formation } \\
\text { Sample No. }\end{array}$} \\
\hline \multicolumn{17}{|c|}{ EL Hanadi } & \multicolumn{6}{|c|}{ DQM } & \multicolumn{4}{|c|}{ EL Mahmiya Mb. } & \\
\hline- & $N$ & $\omega$ & 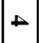 & en & a & $\checkmark x$ & $\infty$ & $\theta$ & $=\bar{N}$ & $\vec{\omega}$ & $\neq$ & $\vec{u}$ & ล & $\checkmark$ & $\bar{\infty} \mid \overline{0}$ & $\frac{1}{0}=$ & $\mathfrak{\Xi}=$ & $N$ & $\mathbf{N}$ & $N$ & $\mathbb{A}$ & 㭊尔 & 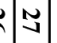 & 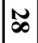 & $\mathfrak{O}$ & $\mathscr{w}$ & \\
\hline $\mathbf{x}$ & $x$ & $>$ & $x$ & $>$ & $>$ & $D$ & $2>1$ & $x=$ & $>>$ & $>x$ & $\Rightarrow$ & $\Omega$ & x] & $>1$ & $\nabla D$ & 2 & $D>$ & $>=$ & $\Rightarrow=$ & x $=$ & x $=$ & $\pi$ & $=$ & a & x & $\pi$ & Abundance \\
\hline 3 & 3 & $\Omega$ & $\Omega$ & $\Omega$ & $\Omega$ & 2 & 20 & $\Omega \leqslant$ & $3 \Omega$ & 23 & 36 & a: & 3 & $\Omega$ & 30 & $2 \leq$ & 39 & a) & 33 & $3 \leq$ & $3 \leq$ & $3=$ & 3 & 3 & 3 & 3 & Preservation \\
\hline & & & & & & & & NP9a & & & & & & & & & & & & & & P9b & & & & & Nannofossil Zone (NP) \\
\hline $\mathbf{F}$ & $\mathbf{F}$ & $\mathbf{F}$ & $\mathbf{R}$ & $\mathbf{F}$ & $\mathbf{F}$ & $\mathbf{R}$ & $\mathbf{F}$ & $\mathbf{F}$ & $\mathbf{R}$ & $\mathbf{F}$ & $\mathbf{F}$ & $\mathbf{R}$ & R & $\mathbf{F}$ & $\mathbf{F}$ & \begin{tabular}{l|l}
$\mathbf{R}$ & $\mathbf{I}$ \\
\end{tabular} & $\mathbf{F}$ & $\mathbf{F}$ & $\mathbf{F}$ & $\mathbf{F}$ & $\mathbf{F}$ & \begin{tabular}{l|l}
$\mathbf{F}$ & $\mathbf{F}$ \\
\end{tabular} & $\mathbf{R}$ & $\mathbf{F}$ & $\mathbf{F}$ & $\mathbf{R}$ & Coccolithus pelagicus \\
\hline $\mathbf{R}$ & $\mathbf{R}$ & VR & & & & $\mathbf{F}$ & & $\mathbf{R}$ & $\mathbf{F}$ & F & & $\mathbf{F}$ & & $\mathbf{R}$ & $\mathbf{F}$ & \begin{tabular}{l|l}
$\mathbf{F}$ & $\mathbf{F}$ \\
\end{tabular} & $\mathbf{F}$ & $\mathbf{F}$ & & $\mathbf{R}$ & & VR & $\mathbf{R}$ & VR & & & Coccolithus eopelagicus \\
\hline $\mathbf{F}$ & $\mathbf{R}$ & $\mathbf{F}$ & $\mathbf{F}$ & $\mathbf{F}$ & $\mathbf{F}$ & $\mathbf{F}$ & $\mathbf{F}$ & \begin{tabular}{|l|l|}
$\mathbf{R}$ & $\mathbf{F}$ \\
\end{tabular} & $\mathbf{F}$ & $\mathbf{F}$ & $\mathbf{F}$ & $\mathbf{F}$ & $\mathbf{F}$ & $\mathbf{F}$ & $\mathbf{F}$ & $\mathbf{F}$ & \begin{tabular}{l|l}
$\mathrm{C}$ & $\mathrm{A}$ \\
\end{tabular} & $\mathbf{A}$ & $\mathbf{C}$ & $\mathbf{F}$ & $\mathbf{F}$ & \begin{tabular}{l|l}
$\mathbf{F}$ & $\mathbf{F}$ \\
\end{tabular} & $\mathbf{F}$ & $\mathbf{R}$ & $\mathbf{R}$ & $\mathbf{R}$ & Ericsonia subpertusa \\
\hline $\mathbf{R}$ & $\mathbf{R}$ & VR & $\mathbf{R}$ & $\mathbf{F}$ & $\mathbf{R}$ & $\mathbf{R}$ & $\mathbf{R}$ & \begin{tabular}{|l|l|} 
VR & $F$ \\
\end{tabular} & $\mathbf{F}$ & $\mathbf{R}$ & & $\mathbf{R}$ & & & & & & $\mathbf{R}$ & \begin{tabular}{l|l}
$\mathbf{R}$ & $\mathbf{V}$ \\
\end{tabular} & $\sqrt{\mathrm{R}}$ & & & $\mathbf{R}$ & & $\mathbf{R}$ & $\mathbf{R}$ & Zygrhablithus bijugatus \\
\hline VR & VR & $R$ & VR & VR & $\mathbf{R}$ & VR & $\mathbf{R}$ & $\mathbf{V R} \mathbf{V}$ & \begin{tabular}{l|ll}
$V R$ & $V R$ \\
\end{tabular} & $\mathbf{R} \mathbf{V R}$ & VR $\mathbf{V}$ & $\mathbf{V R}$ & & VR & $\mathbf{F}$ & $\mathbf{F} \mid \mathbf{v}$ & $\mathbf{V R}$ & & \begin{tabular}{l|l} 
VR & 1 \\
\end{tabular} & $\mathbf{R}$ & & & & & $\mathbf{R}$ & & Thoracosphaera operculata \\
\hline VR & $\mathbf{F}$ & $\mathbf{F}$ & $\mathbf{R}$ & $\mathbf{F}$ & $\mathbf{R}$ & \begin{tabular}{|l|l|}
$\mathbf{F}$ & $\mathbf{F}$ \\
\end{tabular} & \begin{tabular}{l|l}
$\mathbf{F}$ & $\mathbf{F}$
\end{tabular} & \begin{tabular}{|l|l|l}
$\mathbf{R}$ & $\mathbf{F}$ \\
\end{tabular} & \begin{tabular}{l|l}
$\mathbf{R}$ & $\mathbf{F}$ \\
\end{tabular} & & $\mathbf{R}$ & $\mathbf{F}$ & $\mathbf{R}$ & \begin{tabular}{|l|l|l}
$\mathbf{F}$ & $\mathbf{I}$ \\
\end{tabular} & $\mathbf{R}$ & $\mathbf{F}$ & & $\mathbf{F}$ & \begin{tabular}{l|l}
$\mathbf{R}$ & $\mathbf{I}$ \\
\end{tabular} & $\mathbf{F}$ & $\mathbf{R}$ & $\mathbf{R}$ & $\mathbf{R}$ & $\mathbf{R}$ & $\mathbf{R}$ & & Sphenolithus primus \\
\hline $\mathbf{R}$ & $\mathbf{R}$ & $\mathbf{R}$ & $\mathbf{R}$ & $\mathbf{F}$ & $\mathbf{R}$ & $\mathbf{R}$ & \begin{tabular}{l|l|}
$\mathbf{R}$ & $\mathbf{F}$ \\
\end{tabular} & & $\mathbf{R}$ & & $\mathbf{R}$ & $\mathbf{R}$ & & $\mathbf{R}$ & $\mathbf{R}$ & \begin{tabular}{l|l}
$\mathbf{R}$ & $\mathbf{F}$ \\
\end{tabular} & $\mathbf{R}$ & & \begin{tabular}{l|l}
$\mathbf{V R}$ & $\mathbf{V}$ \\
\end{tabular} & \begin{tabular}{l|l}
$\mathbf{R}$ & $\mathbf{F}$ \\
\end{tabular} & \begin{tabular}{l|l}
$\mathbf{R}$ & $\mathbf{V}$ \\
\end{tabular} & VR VR & $\begin{array}{ll}\mathbf{R} & \mathbf{F} \\
\end{array}$ & $\mathbf{R}$ & $\mathbf{R}$ & & Discoaster multiradiatus* \\
\hline VR & & $\mathbf{R}$ & $\mathbf{R}$ & $\mathbf{F}$ & $\mathbf{R}$ & & $\mathbf{R}$ & & $\mathbf{R}$ & & & $\mathbf{R}$ & & $\mathbf{R}$ & $\mathbf{R}$ & \begin{tabular}{l|l}
$\mathbf{R}$ & $\mathbf{F}$ \\
\end{tabular} & $\mathbf{R}$ & & & & & & & & & & Discoaster lenticularis \\
\hline VR & & $\mathbf{R}$ & $\mathbf{R}$ & $\mathbf{R}$ & $\mathbf{R}$ & $\mathbf{R}$ & \begin{tabular}{l|l|}
$\mathbf{R}$ & $\mathbf{R}$ \\
\end{tabular} & $\mathbf{R}$ & $\mathbf{R}$ & & $\mathbf{R}$ & $\mathbf{R} \mathbf{Y}$ & $\mathbf{V R}$ & $\mathbf{F}$ & VF & \begin{tabular}{l|l}
$\mathbf{R}$ & $\mathbf{F}$ \\
\end{tabular} & $\mathbf{R}$ & & & \begin{tabular}{l|l}
$\mathbf{R}$ & $\mathbf{F}$ \\
\end{tabular} & $\mathbf{R}$ & & VR & $\mathbf{R}$ & $\mathbf{R}$ & & Toweius pertusus \\
\hline $\mathbf{R}$ & & $\mathbf{R}$ & $\mathbf{R}$ & & VR & & & & & & & $\mathbf{R}$ & $\mathbf{R}$ & & & & & & & & & & & & & & Toweius tovae \\
\hline $\mathbf{R}$ & VR & F & $\mathbf{F}$ & $\mathbf{F}$ & $\mathbf{F}$ & $\mathbf{F}$ & $\mathbf{F}$ & $\mathbf{R}$ & & $\mathbf{R}$ & & $\mathbf{V R}$ & $\mathbf{R}$ & & VF & $\mathbf{R}$ & & & $\mathbf{V R}$ & & & VR VR & & $\mathbf{R}$ & $\mathbf{R}$ & $\mathbf{R}$ & Fasciculithus tympaniformis * \\
\hline VR & & $\mathbf{R}$ & VR & $\mathbf{R}$ & & VR & $\mathbf{R}$ & \begin{tabular}{l|l} 
VR & F \\
\end{tabular} & $\mathbf{R}$ & $\mathbf{R}$ & $\mathbf{R}$ & $\mathbf{R}$ & & $\mathbf{R}$ & & & & & & & & & & & & & Fasciculithus schaubii \\
\hline VR & & $\mathbf{R}$ & $\mathbf{R}$ & $\mathbf{R}$ & $\mathbf{R}$ & \begin{tabular}{|l|l} 
& $\mathbf{R}$ \\
\end{tabular} & $\mathbf{R}$ & & VR & $\mathbf{R}$ & & & & & & & & & & & & & & & & & Fasciculithus bobii \\
\hline $\mathbf{R}$ & & $\mathbf{F}$ & & $\mathbf{F}$ & $\mathbf{F}$ & $\mathbf{R}$ & \begin{tabular}{|l|l|}
$\mathbf{R}$ & $\mathbf{R}$ \\
\end{tabular} & \begin{tabular}{|l|l} 
VR & F \\
\end{tabular} & \begin{tabular}{l|l|}
$\mathbf{R}$ & $\mathbf{R}$ \\
\end{tabular} & $\mathbf{R}$ & $\mathbf{R}$ & $\mathbf{R}$ & $\mathbf{R}$ & $\mathbf{V R}$ & & & $\mathbf{R}$ & & $\mathbf{V R} \mathbf{V}$ & $\mathbf{N R}$ & & & VR & VR & $\mathbf{R}$ & $\mathbf{R}$ & Fasciculithus involutus \\
\hline $\mathbf{R}$ & $\mathbf{F}$ & $\mathbf{F}$ & & $\mathbf{F}$ & $\mathbf{F}$ & $\mathbf{R}$ & \begin{tabular}{|l|l|l}
$\mathbf{R}$ & $\mathbf{T}$ \\
\end{tabular} & \begin{tabular}{|l|l|l|l} 
VR & F \\
\end{tabular} & \begin{tabular}{l|l|}
$\mathbf{R}$ & $\mathbf{F}$ \\
\end{tabular} & $\mathbf{R}$ & & & $\mathbf{R}$ & & $\mathrm{VF}$ & \begin{tabular}{l|l}
$\mathbf{R}$ & $\mathbf{V}$ \\
\end{tabular} & VR & & & $\sqrt{\mathbf{R}}$ & & & VR & & & & Zygolithus sheldoniae \\
\hline & & $\mathbf{R}$ & & & & & \begin{tabular}{l|l|}
$\mathbf{R}$ & \\
\end{tabular} & & & & $\mathrm{VR}$ & & $\mathbf{V R}$ & & & & & & & & $\mathbf{R}$ & & & & & & Toweius eminens \\
\hline & & VR & & VR & VR & \begin{tabular}{|l|l|}
$\mathbf{R}$ & \\
\end{tabular} & $\mathrm{VR}$ & \begin{tabular}{|l|l}
$\mathbf{R}$ & $\mathbf{V}$ \\
\end{tabular} & \begin{tabular}{l|l}
$V R$ & $V R$ \\
\end{tabular} & R VR & & & & VR & VR VF & \begin{tabular}{l|l}
$\mathbf{R}$ & $\mathbf{V}$ \\
\end{tabular} & $\mathbf{V R}$ & & & & \begin{tabular}{l|l|}
$\mathbf{R}$ & $\mathbf{V}$ \\
\end{tabular} & VR VR & $\mathbf{R} \mathbf{V R}$ & & & $\mathbf{R}$ & Campylosphaera eodela \\
\hline & & $\mathbf{R}$ & & $\mathbf{F}$ & $\mathbf{F}$ & \begin{tabular}{|l|l|}
$\mathbf{F}$ & $\mathbf{R}$ \\
\end{tabular} & \begin{tabular}{|l|l|}
$\mathbf{R}$ & $\mathbf{R}$ \\
\end{tabular} & \begin{tabular}{|l|l|}
$\mathbf{R}$ & $\mathbf{F}$ \\
\end{tabular} & \begin{tabular}{l|l}
$\mathbf{R}$ & $\mathbf{F}$ \\
\end{tabular} & $\mathbf{R}$ & & $\mathbf{V R}$ & $\mathbf{R}$ & & F & \begin{tabular}{l|l}
$\mathbf{F}$ & $\mathbf{F}$ \\
\end{tabular} & $\begin{array}{ll}\mathbf{R} & \mathbf{R} \\
\end{array}$ & $\mathbf{R}$ & & & & & VR & & $\mathbf{R}$ & & Zygodiscus plectopons \\
\hline & & VR & & VR & VR & \begin{tabular}{|l|l} 
VR & VI \\
\end{tabular} & \begin{tabular}{l|l}
$\mathbf{R}$ & \\
\end{tabular} & VR & & & & $\mathbf{V R}$ & & $\mathbf{V R}$ & $\mathbf{R}$ & \begin{tabular}{r|r}
$\mathbf{R}$ \\
\end{tabular} & & $\mathbf{R}$ & & & & & & & & & Calcisolenia aperta \\
\hline & & & & VR & & VR & & & \begin{tabular}{l|l}
$\mathbf{V R}$ & \\
\end{tabular} & VR & VR V & $\mathbf{V R}$ & $\mathbf{R}$ & & $\mathbf{R}$ & $\mathrm{R}$ & & & & \begin{tabular}{l|l}
$\mathbf{R}$ & $\mathbf{V}$ \\
\end{tabular} & $\begin{array}{ll}\mathbf{R} & \mathbf{V} \\
\end{array}$ & $\sqrt{R}$ & VR & $\mathbf{R}$ & $\mathbf{R}$ & $\mathbf{R}$ & Ellipsolithus macellus* \\
\hline & & & & VR & & & VR & & \begin{tabular}{l|l}
$\mathbf{R}$ & $\mathbf{V R}$ \\
\end{tabular} & $\begin{array}{ll}\mathbf{R} \\
\end{array}$ & & $\mathbf{R} \mathbf{Y}$ & $\mathbf{V R}$ & & & & & & & & & & VR & & & & Braarudosphaera bigelowii \\
\hline & & & & VR & VR & & $\mathrm{VR}$ & & VR & $\overline{\mathbf{R}}$ & $\mathrm{VR}$ & $\overline{V R}$ & $\mathbf{V R}$ & & & & & & & & & & & & & & Ellipsolithus distichus \\
\hline & & & & $\mathrm{VR}$ & & & & & & & & & & & & & & & & & & & & & & & Discoaster mohleri * \\
\hline & & & & $\mathrm{VR}$ & VR & $\mathbf{R}$ & VR & & & & & & & & & & & & & & & & & & & & Chiasmolithus bidens \\
\hline & & & & $\mathbf{R}$ & & \begin{tabular}{l|l} 
VR & $R$ \\
\end{tabular} & \begin{tabular}{|l|l|}
$\mathbf{R}$ & $\mathbf{R}$ \\
\end{tabular} & & $\mathbf{R}$ & 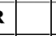 & & & & & & & & & & & & & & & & & Fasciculithus richardii \\
\hline & & & & & $\mathbf{R}$ & \begin{tabular}{|l|l|}
$\mathbf{R}$ & \\
\end{tabular} & $\mathbf{R}$ & & VR & $\mathbf{R}$ & $\mathrm{VR}$ & & & VR & & & & & $\mathbf{V R}$ & & & & & & & & Fasciculithus clinatus \\
\hline & & & & & VR & VR & & & & & & & & & & & & & & & & & & & & & Discoaster falcatus \\
\hline & & & & & $\mathbf{R}$ & \begin{tabular}{|l|l|}
$\mathbf{R}$ & $\mathbf{R}$ \\
\end{tabular} & \begin{tabular}{l|l}
$\mathbf{R}$ & $\mathbf{R}$ \\
\end{tabular} & & $\mathbf{R}$ & & $\mathbf{R}$ & $\mathbf{R}$ & $\mathbf{R}$ & & & & $\mathbf{R}$ & & & & & & & & & & Zygodiscus adamas \\
\hline & & & & & $\mathbf{R}$ & & & & & & & & & & & & & $\mathbf{R}$ & & & & & & & & & Thoracosphaera saxea \\
\hline & & & & & VR & & & & VR & & & & & & & & & & & & & & & & & & Bomolithus megastypus \\
\hline & & & & & & VR & VR & & \begin{tabular}{l|l}
$\mathrm{VR}$ & $\mathrm{VR}$ \\
\end{tabular} & $\mathbf{R}$ & VR V & $\mathbf{V R}$ & & & VF & $\mathbf{R}$ & & & & & $\mathbf{R}$ & & VR & $\mathbf{R}$ & & $\mathbf{R}$ & Chiasmolithus consuetus \\
\hline & & & & & & & $\mathbf{R}$ & $\mathbf{R}$ & \begin{tabular}{l|l}
$\mathbf{R}$ \\
\end{tabular} & & & & & & & & & & & & & & & & & & Pontosphaera exilis \\
\hline & & & & & & & $\mathbf{R}$ & & & & & & $\mathbf{R}$ & $\mathbf{V R}$ & VF & $\mathbf{R}$ & & $\mathbf{V R}$ & $\mathbf{V R}$ & & & VR VR & $\begin{array}{ll}\mathbf{R} & \mathbf{F} \\
\end{array}$ & & & $\mathbf{F}$ & Neochiastozygus junctus \\
\hline & & & & & & & & $\mathbf{R}$ & & & & & & & & & \begin{tabular}{l|l}
$\mathbf{R}$ & $\mathbf{V} 1$ \\
\end{tabular} & VR & & & & & & & & & Ericsonia robusta \\
\hline & & & & & & & & & VR & $\begin{array}{ll}\mathbf{R} & \mathbf{R} \\
\end{array}$ & & & $\mathbf{R}$ & & & & & & & & & & & & & & Pontoshphera pulchra \\
\hline & & & & & & & & & VR & & & $\mathbf{R} \mathbf{Y}$ & VR & & & & & & & & & & & & & & Pontosphaera formosa \\
\hline & & & & & & & & & & $\mathbf{R}$ & & & & $\mathbf{F}$ & & & & & & & & & VR & & & & Pontosphaera ocellata \\
\hline & & & & & & & & & & & VR & & & $\mathbf{V R}$ & & & & & $\mathbf{V R}$ & & & & & & & & Neococcolithes protenus \\
\hline & & & & & & & $\pi \|$ & 28 & & & & & & $\mathbf{R}$ & & & & & & & & & & & & & Pontosphaera plana \\
\hline & & & & & 3 & & & 2 & & & & & & VR & & & & & & & & & & & & & Discoaster backmanii \\
\hline & & & & & $=\frac{1}{4}$ & $\cos ^{2}$ & & 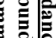 & & & & & & & VR & & $\mathbf{V R}$ & & & $\mathbf{N R}$ & & & & & & & Lophodolithus nascens \\
\hline & & & & & $\bar{E}_{0}^{2}$ & $\pi$ & 药 & $=$ & & & & & & & & & VR & & $\mathbf{V R}$ & & & & & & & & Discoaster araneus * \\
\hline & & & & & : & & & & & & & & & & & & VR & & VR & & & & & & & & Rhomboaster bitrifida* \\
\hline & & & & & 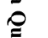 & & & & & & & & & & & & $\mathbf{V R}$ & & & & & & & & & & Discoaster anartios * \\
\hline & & & & & $\stackrel{\bar{E}}{=}$ & & & iil & & & & & & & & & \begin{tabular}{l|l}
$\mathbf{F}$ & $\mathbf{R}$ \\
\end{tabular} & $\mathbf{R}$ & & & & & & & & & Ericsonia universa \\
\hline & & & & & $\frac{7}{3}$ & & & 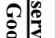 & & & & & & & & & & & $\mathbf{V R}$ & & & & & & & & Discoaster paelikei \\
\hline & & & & & $\frac{9}{E}$ & & $\frac{2}{2}$ & ¿. & & & & & & & & & & & & & & & VR & & $\mathbf{V R}$ & \begin{tabular}{|l|l|} 
VR \\
\end{tabular} & Rhomboaster cuspis* \\
\hline & & & & & $\stackrel{\vec{\sigma}}{\dddot{q}}$ & & & & & & & & & & & & & & & & & & VR & & & $\mathbf{R}$ & Pontosphaera multipora \\
\hline & & & & & & & & & & & & & & & & & & & & & & & VR & & & & Discoaster mahmoudii ${ }^{*}$ \\
\hline
\end{tabular}

Figure 5. Stratigraphic distribution of calcareous nannofossil species in Naqb Assiut section, Kharga Oasis, Western Desert, Egypt.

The two subzones of AUBRY (1999) were identified in our study. The NP9a/NP9b is marked by the LO of Discoaster araneus at the Gabal Umm El Ghanayim section (Fig. 4) and the LOs of Rhomboaster bitrifida, Discoaster araneus and D. anartios can be used to delineate the base of NP9b at the Gabal Naqb Assiut section (Fig. 5).

The total range of D. mahmoudii characterizes the subzone NP9c (AUBRY \& SALEM, 2012). In the Umm El Ghanayim sec- tion, D. mahmoudii first appears in sample 37. The LO of $R$. spineus in sample 34, is restricted to NP9c. We can conclude that the Subzone NP9c is approximately represented by a short interval (samples 34-38) in this section. In the Naqb Assiut section, the LOs of $D$. araneus, $D$. anartios and $R$. bitrifida (markers of NP9b) occur in sample 20. Nevertheless, D. mahmoudii appears only in sample 27 and the base of the NP9c can also be tentatively placed at this sample. 


\subsubsection{Tribrachiatus contortus Zone (NP10)}

Definition: This biostratigraphic interval is defined by the LO of Tribrachiatus bramlettei or Discoaster diastypus at its base and the HO of T. contortus at its top.

Authors: HAY (1964)

Age: Early Eocene

Correlation: Subzones Campylosphaera eodela (CP8b) and Tribrachiatus contortus (CP9a) of OKADA \& BUKRY (1980). It is equivalent to Fasciculithus tympaniformis TZ (CNE1), to Toweius eminens PRZ (CNE2) and to the lowermost part of Tribrachiatus orthostylus BZ (CNE3) of AGNINI et al. (2014) (Table 2).

Occurrence: At the Gabal Umm El Ghanayim section, $T$. bramlettei has rare occurrences and the top of the zone cannot be defined due to the complete absence of Tribrachiatus contortus.

Remarks on assemblages: A new species, Tribrachiatus digitalis, was described from the DSDP Site 550, and used to subdivide the NP10 Zone into four subzones (AUBRY, 1996). The LO of T. bramlettei defines the base of the NP10a Subzone, the LO of T. digitalis defines the base of the NP10b Subzone, the HO of T. digitalis defines the base of the NP10c Subzone, and the LO of $T$. contortus defines the base of the NP10d Subzone. The base of the NP11 Zone (= top of NP10d Subzone) is defined by the HO of $T$. contortus.

AUBRY et al. (1999) also used this subdivision in their description of the Esna Shale of Egypt (Gebel Qreiya, Gebel Kilabiya). DUPUIS et al. (2003) used the same four nannofossil subzones for dividing the NP10 of MARTINI (1971) at the Dababiya section.

Only the subzones NP10a and NP10b have been identified at the Gabal Umm El Ghanayim section. In the Umm El Ghanayim section, the NP10a Subzone includes the interval from sample 39 to 40 . The interval from samples 41 to 48 can be assigned to the NP10b Subzone, based on the observed first rare occurrence of T. digitalis.

\section{DISCUSSION}

\subsection{Palaeocene/Eocene (P/E) boundary}

The onset of the carbon isotopic excursion (CIE) associated with the Palaeocene/Eocene Thermal Maximum (PETM) is considered as a best criterion for defining the $\mathrm{P} / \mathrm{E}$ boundary in marine and non-marine sequences worldwide.

Table 3. Calcareous nannofossil bioevents at the Palaeocene/Eocene boundary recorded by different authors in the Eastern and Western Desert and Nile Valley.

\begin{tabular}{|c|c|c|c|c|}
\hline Sections & Authors & Location & P/E boundary & Calcareous nannofossil bioevents \\
\hline Taramsa & \multirow{4}{*}{ YOUSSEF (2016) } & West Qena & \multirow{4}{*}{ NP9a/NP9b } & \multirow{4}{*}{$\begin{array}{l}\text { LOs of D. araneus, D. anartios, D. aegyptiacus, } \\
\text { Rhomboaster spp., Ca. eodela/dela }\end{array}$} \\
\hline & & Quseir & & \\
\hline Qeryia & & Wadi Qena & & \\
\hline Arras & & Safaga & & \\
\hline Darb Gaga-1 & \multirow{3}{*}{$\begin{array}{l}\text { FARIS \& ABDEL SABOUR } \\
\text { (a; b; c) (in prep) }\end{array}$} & \multirow{2}{*}{$\begin{array}{l}\text { Southern Kharga } \\
\text { Oasis }\end{array}$} & NP9a/NP9b & $\begin{array}{l}\text { LOs of D. araneus, D. anartios, } \\
\text { R. intermedia }\end{array}$ \\
\hline Darb Gaga-2 & & & NP9a/NP9b & $\begin{array}{l}\text { LOs of } D \text {. araneus, D. anartios, } \\
\text { R. bitrifida }\end{array}$ \\
\hline AL Aguz area & & $\begin{array}{c}\text { Northern Kharga } \\
\text { Oasis }\end{array}$ & NP9a/NP9b & LOs of $R$. calcitrapa, R. spineus, D. araneus, D. anartios \\
\hline Nag El-Quda & Youssef (2015) & Eastern Desert & NP9a/NP9b & LAD of D. araneus and Rhomboaster spp. \\
\hline Gebel Ghanima & $\begin{array}{l}\text { KHALIL \& AL } \\
\text { SAWY (2014) }\end{array}$ & Kharga Oasis & NP9a/NP9b & $\begin{array}{l}\text { LO of R. cuspis } \\
\text { Abrupt decrease of Fasciculithus spp. }\end{array}$ \\
\hline El Serai & \multirow{2}{*}{$\begin{array}{l}\text { TANTAWY } \\
(2006)\end{array}$} & East Qena & \multirow{2}{*}{ NP9a/NP9b } & LOs of Ca. eodela/dela, D. araneus \\
\hline Taramsa & & West Qena & & Abrupt decrease of Fasciculithus spp. \\
\hline Geirya (Abu Had) & \multirow{2}{*}{ AUBRY (1999) } & East Qena & NP9a/NP9b & \multirow{2}{*}{$\begin{array}{l}\text { LOs of D. araneus, R. spineus, } \\
\text { R. cuspis, D.araneus }\end{array}$} \\
\hline Kilabiya & & Nile Valley & NP9a/NP9b & \\
\hline Teir/Tarawan & \multirow{5}{*}{ FARIS et al. (1999) } & \multirow{2}{*}{ Kharga Oasis } & \multirow{3}{*}{ within NP9 } & HOs of Fasciculithus spp. \\
\hline Um El-Ghanayim & & & & LOs of T. bramlettei, D. binodosus \\
\hline El Sheikh Eissa & & East Qena & & HOs of Fasciculithus spp. \\
\hline El-Homra El-Shanka & & \multirow{2}{*}{ Esna } & \multirow{2}{*}{ NP9/NP10 } & LO of T. bramlettei \\
\hline G. El-Shaghab & & & & LO of T. bramlettei \\
\hline North Gunna & \multirow{2}{*}{ FARIS \& STROUGO (1998) } & \multirow{2}{*}{ Nile Valley } & NP9/NP10 & \multirow{2}{*}{$\begin{array}{l}\text { HOs of F. tympaniformis, F. bobii, F. clinatus, F. clinatus, } \\
\text { F. involutus, D. mediosus }\end{array}$} \\
\hline El Sheikh Marzouk & & & NP9/NP10 & \\
\hline Duwi & \multirow{4}{*}{ TANTAWY (1998) } & Quseir & NP9a/NP9b & \multirow{4}{*}{ LO of Ca. eodela/dela } \\
\hline Oweina & & Nile Valley & NP9a/NP9b & \\
\hline Abu Had & & NE Desert & NP9a/NP9b & \\
\hline Wadi Tarfa & & East Qena & NP9a/NP9b & \\
\hline $\begin{array}{l}\text { Wadi El } \\
\text { Dakhl }\end{array}$ & $\begin{array}{l}\text { STROUGO \& } \\
\text { FARIS (1993) }\end{array}$ & $\begin{array}{l}\text { Southern Galala } \\
\text { plateau }\end{array}$ & NP9/NP10 & LO of T. bramlettei \\
\hline $\begin{array}{l}\text { Abu Had } \\
\text { El Serai } \\
\text { Tarmsa }\end{array}$ & $\begin{array}{l}\text { FARIS et al. } \\
\text { (1989) }\end{array}$ & Qena Region & $\begin{array}{l}\text { NP9/NP10 } \\
\text { top NP9 } \\
\text { top NP9 }\end{array}$ & $\begin{array}{l}\text { LO of D.mahmoudii - HOs of F.alanii, } \\
\text { F. bobii, D. mohleri, D. falcatus, F. tympaniformis }\end{array}$ \\
\hline Wasif area & $\begin{array}{l}\text { HEWAIDY \& } \\
\text { FARIS (1989) }\end{array}$ & Safaga District & NP9/NP10 & $\begin{array}{l}\text { LOs of } T \text {. nunnii, D. diastypus } \\
\text { HO of } F \text {. tympaniformis }\end{array}$ \\
\hline Um el Huetat & FARIS (1988b) & & NP9/NP10 & HOs of F. alanii, F.tonii, F. tympaniformis \\
\hline
\end{tabular}

$\mathrm{LO}=$ Lowest Occurrence; $\mathrm{HO}=$ Highest Occurrence; $\mathrm{HA}=$ Highest Appearance; $\mathrm{LAD}=$ Lowest Appearance Datum 
At the Dababiya section (the Global Standard Stratotype section and Point - GSSP) the base of the lower Eocene beds $(73 \mathrm{~cm}$ thick) is carbonate free and barren of calcareous nannofossils, and the taxa Discoaster araneus, Discoaster anartios, Rhomboaster spineus, and Rhomboaster spp. were recorded directly above this barren interval (DUPUIS et al., 2003).

El DAWY et al. (2016) studied the planktonic foraminifera at the Naqb Assiut section. They observed an irregular unconformity surface associated with pebbles and bioturbated sand at the Palaeocene/Eocene boundary interval, and they suggested the existence of a minor hiatus at this boundary. In the present study, this small hiatus cannot be detected by means of nannofossils.

At the Wadi Nukhul section, West Central Sinai, KHOZYEM et al. (2013), suggest that the lowermost Eocene sediments are absent. The presence of a short hiatus at the P/E boundary is also observed in other sections from Egypt (Gabal Duwi and G. Aweina sections; SPEIJER et al., 2000). The presence of a hiatus at the P/E boundary was recorded at the W. Nukhul, Wadi Matulla, and G. Mekattub sections (West Central Sinai) (FARIS et al., 2015b).

At the Gabal Umm El Ghanayim section, the LO of Discoaster araneus was used to delineate the base of Subzone NP9b, while the LOs of Rhomboaster bitrifida, Discoaster araneus and $D$. anartios can be used for approximation of the NP9a/NP9b subzonal boundary (early Eocene) at Gabal Naqb Assiut section.

\subsection{Calcareous nannofossil bioevents}

Fasciculithus alanii occurs below the P/E boundary, and is restricted to the NP9a subzone (DUPUIS et al., 2003). The HO of this species has also been reported in several areas in Egypt such as Gabal Aweina, Gabal Duwi, Gabal Abu Had (AUBRY, 1998;
VON SALIS et al., 1998; AUBRY \& SALEM, 2013) and at Wadi Nukhul in West Central Sinai (KHOZYEM et al., 2013). The HO of $F$. alanii at the Markha section (WC Sinai), can be used to approximate the $\mathrm{P} / \mathrm{E}$ boundary (FARIS \& FAROUK, 2015). In the present study, the $\mathrm{HO}$ of Fasciculithus alanii was recorded at the base of the NP9b Subzone (sample 27) at the Gabal Umm El Ghanayim section.

The first occurrence of Discoaster mahmoudii is noticed in the uppermost part of the NP9b Subzone in the investigated sections (Figs. 4, 5), and has its last appearance (HO in sample 39) in the lower part of the NP10a Subzone at the Gabal Umm El Ghanayim section.

The lowest occurrence of Campylosphaera eodela is within the NP9a Subzone (late Palaeocene) at the Gabal Umm El Ghanayim section, indicating that this species cannot be used as a reliable marker for subdividing the CP8 (=NP9) of OKADA \& BUKRY (1980) into CP8a and CP8b (=NP9a and NP9b). The calcareous nannofossil bioevents, recorded by different authors in many sections in Egypt, are summarized in Tables 3 and 4.

\subsection{Calcareous nannofossil turnover}

The calcareous nannofossil assemblages displayed turnover at the P/E boundary transition (Figs. 4, 5). Several species became extinct, others continued into the Eocene and some new taxa appeared in the Eocene. Some Fasciculithus (Fasciculithus tonii, F. richardii, F. schaubii. F. thomasii, F. clinatus, F. lillianiae) and Discoaster species (D. mediosus, D. mohleri) disappeared within the NP9a Subzone. The coccoliths that survived include Campylosphaera eodela, Discoaster lenticularis, D. multiradiatus, Fasciculithus tympaniformis, F. involutus and Zygrhablithus bijugatus.

Table 4. Calcareous nannofossil bioevents at the Palaeocene/Eocene boundary recorded by different authors in Sinai.

\begin{tabular}{|c|c|c|c|c|}
\hline Sections & Authors & Location & P/E boundary & Calcareous nannofossil bioevents \\
\hline Abu Zenima & BOLLE et al. (2000) & Western Sinai & wihin NP9 & C. pelagicus acme \\
\hline Bir & FARIS et al. (2005) & \multirow{6}{*}{ 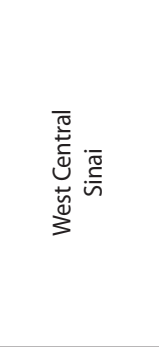 } & NP9a/NP9b & HA of Fasciculithus spp. \\
\hline El Markha & FARIS \& FAROUK (2015) & & NP9a/NP9b & $\begin{array}{l}\text { Co. bownii acme } \\
\text { LOs of } R \text {. bitrifida, R. intermedia } \\
\text { HO of F. alanii }\end{array}$ \\
\hline \multirow{4}{*}{ Matulla } & ABU SHAMA et al. (2007) & & NP9a/NP9b & $\begin{array}{l}\text { LOs of } R \text {. cuspis, } R \text {. calcitrapa, } \\
D . \text { mahmoudii, D. araneus }\end{array}$ \\
\hline & BOLLE et al. (2000) & & wihin NP9 & LOs of $D$. araneus, $R$. bitrifida, $R$. intermedia \\
\hline & FARIS et al. (2015) & & NP9a/NP10 & LO of T. bramlettei \\
\hline & FARIS \& FAROUK (2015) & & NP9a/NP9b & LO of R. intermedia \\
\hline \multirow{3}{*}{ Nukhul } & FARIS \& SALEM (2007) & \multirow{3}{*}{ 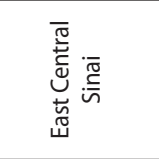 } & NP9a/NP9b & LOs of R. intermedia, R. calcitrapa \\
\hline & KHOZYEM et al. (2013) & & NP9a/NP9b & LOs Ca. eodela/dela, R. cuspis \\
\hline & FARIS et al. (2015) & & NP9a/NP10 & $\begin{array}{l}\text { LOs of T. bramlettei, D. anartios, D. araneus, R. bitrifida, R. cuspis, } \\
\text { R. intermedia, R. spineus }\end{array}$ \\
\hline Mukattab & FARIS et al. ( 2015) & \multirow{3}{*}{ 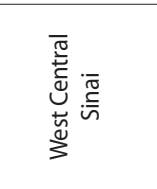 } & NP9a/NP10 & $\begin{array}{l}\text { LOs T. bramlettei, D. anartios, D. araneus, R. bitrifida, R. cuspis, } \\
\text { R. intermedia, R. spineus }\end{array}$ \\
\hline \multirow[b]{2}{*}{ Wadi Feiran } & FARIS \& SALEM ( 2007) & & NP9a/NP9b & LO of $R$. solus \\
\hline & FARIS et al. (2015) & & NP9a/NP10 & $\begin{array}{l}\text { LOs T. bramlettei, D. anartios, D. araneus, R. bitrifida, R. cuspis, } \\
\text { R. intermedia, R. spineus }\end{array}$ \\
\hline El Mishiti & FARIS \& ABU SHAMA (2007) & EC Sinai & NP9/NP10 & LOs of R. cuspis, R. calcitrapa, D. mahmoudii, D. araneus \\
\hline $\begin{array}{l}\text { Muwaylih } \\
\text { Amr }\end{array}$ & AYYAD et al. (2003) & NE Sinai & NP9a/NP9b & $\mathrm{HO}$ of F. alanii \\
\hline El Burk area & FARIS \& ZAHRAN (2002) & NC Sinai & NP9/NP10 & LO of T. bramlettei \\
\hline El Sheikh Attya & LUNING et al. (1998) & EC Sinai & NP9/NP10 & $\begin{array}{l}\text { LOs of } D \text {. diastypus, } D \text {. binodosus } \\
\text { HOof } F \text {. tympaniformis }\end{array}$ \\
\hline $\begin{array}{l}\text { El Ain } \\
\text { El Falig }\end{array}$ & FARIS (1988a) & NE Sinai & NP9/NP10 & $\begin{array}{l}\text { LOs of T. contortus, D. diastypus, } \\
\text { D. mahmoudii }\end{array}$ \\
\hline
\end{tabular}


The Eocene assemblage includes Rhomboaster taxa ( $R$. intermedia, $R$. bitrifida, $R$. cuspis, and $R$. spineus) in addition to the first appearances of Discoaster araneus, D. anartios, D. paelikei, and D. mahmoudii which occurred within the NP9b Subzone (early Eocene).

In the present study, the Rhomboaster-Discoaster taxa did not occur simultaneously, having very rare relative abundances (Figs. 4, 5). The first appearance of $D$. araneus is at the base of $\mathrm{NP} 9 \mathrm{~b}$ at the Um El Ghanayim section. Other taxa occurred in sequential order: D. anartios, R. bitrifida, R. cuspis and $R$. spineus. At the Gabal Naqb Assiut section, D. araneus, D. anartios and R. bitrifida co-occurred at the base of the NP9b Subzone, and R. cuspis appeared later at a higher level within NP9b.

\subsection{Diversity}

The diversity of calcareous nannofossils is the number of species in each sample. It reaches its maximum in the late Palaeocene (PERCH-NIELSEN, 1985; FARIS \& SALEM, 2007). The calcareous nannofossil diversity reaches a maximum of 26 species in the NP9a Subzone (sample no. 17), with 21 species in NP9b at G. Umm El Ghanayim section. Above this subzone, fluctuations in species diversity occur, with variations from 24 to 7 species in the NP10 Zone with a generally decreasing trend.

At the Naqb Assiut section, the number of species fluctuates within NP9a from 5 to a maximum of 23 species (sample no 6), and reaches about 16 species in the upper part of late Palaeocene, while above that level the diversity fluctuates from 7 to 18 species in the NP9b Subzone (early Eocene).
The Fasciculithus diversity decreases sharply within the NP9b Subzone and it disappears completely shortly in the lowermost part of NP10 (Fig. 4). In the basal part of Zone NP10, $F$. tympaniformis and $F$. involutus were recorded. The same observations were recorded in the Qreiya, Duwi and Oweina sections (VON SALIS et al., 1998), in G. Taramsa, G. Serai (TANTAWY, 2006) and in the Gabal Qreiya, Gabal Arras, and Gabal Duwi sections (YOUSSEF, 2009, 2015, 2016).

\subsection{Palaeoecology}

Several authors have proposed a link between some nannofossil species abundance and oceanographic changes that may have occurred during the PETM (e.g. BRALOWER, 2002; KAHN \& AUBRY, 2004; TREMOLADA \& BRALOWER, 2004; GIBBS et al., $2006 \mathrm{a}, \mathrm{b}$; JIANG \& WISE, 2006). Several nannofossil taxa are known to be adapted to warmer, more oligotrophic surface water environments. The genus Discoaster has often been associated with warm water species (EDWARDS, 1968; BUKRY, 1973), while AUBRY (1992) proposed that this genus was also adapted to oligotrophic conditions. The genera Fasciculithus and Sphenolithus were adapted to warmer, more oligotrophic environments, as noted in different ODP sites (BRALOWER, 2002; GIBBS et al., 2006a, b). Taxa belonging to the Rhomboaster-Tribrachiatus lineage have been regarded as proxies for warm-waters and oligotrophic conditions (AUBRY, 1998; BRALOWER, 2002; TANTAWY, 2006; MUTTERLOSE et al., 2007).

Coccolithus pelagicus is a dominant species of the high latitudes from the Neogene to the present, based on palaeobiogeo-

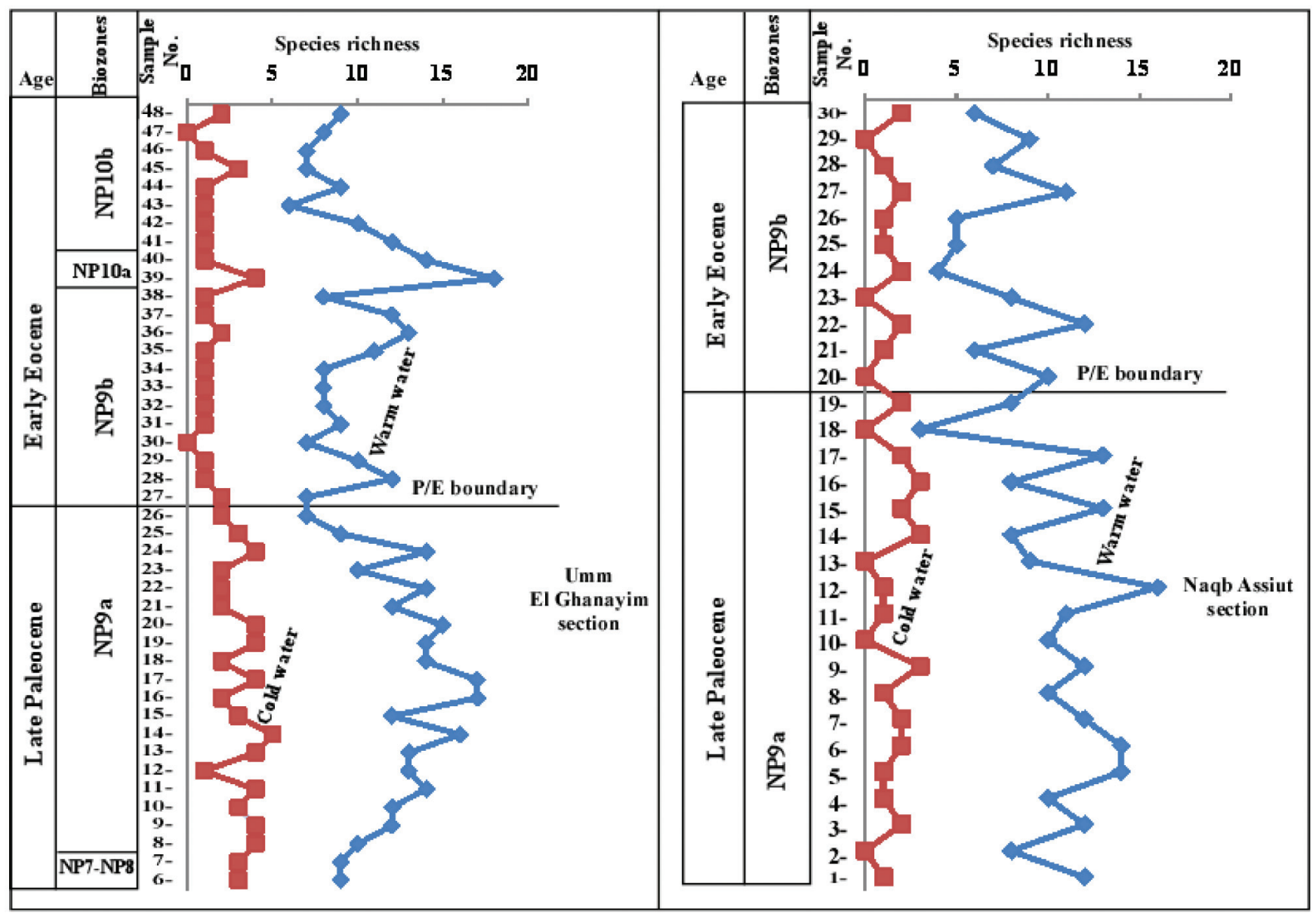

Figure 6. Vertical variations in the number of warm water and cold water species in Umm El Ghanayim and Naqb Assiut sections. 


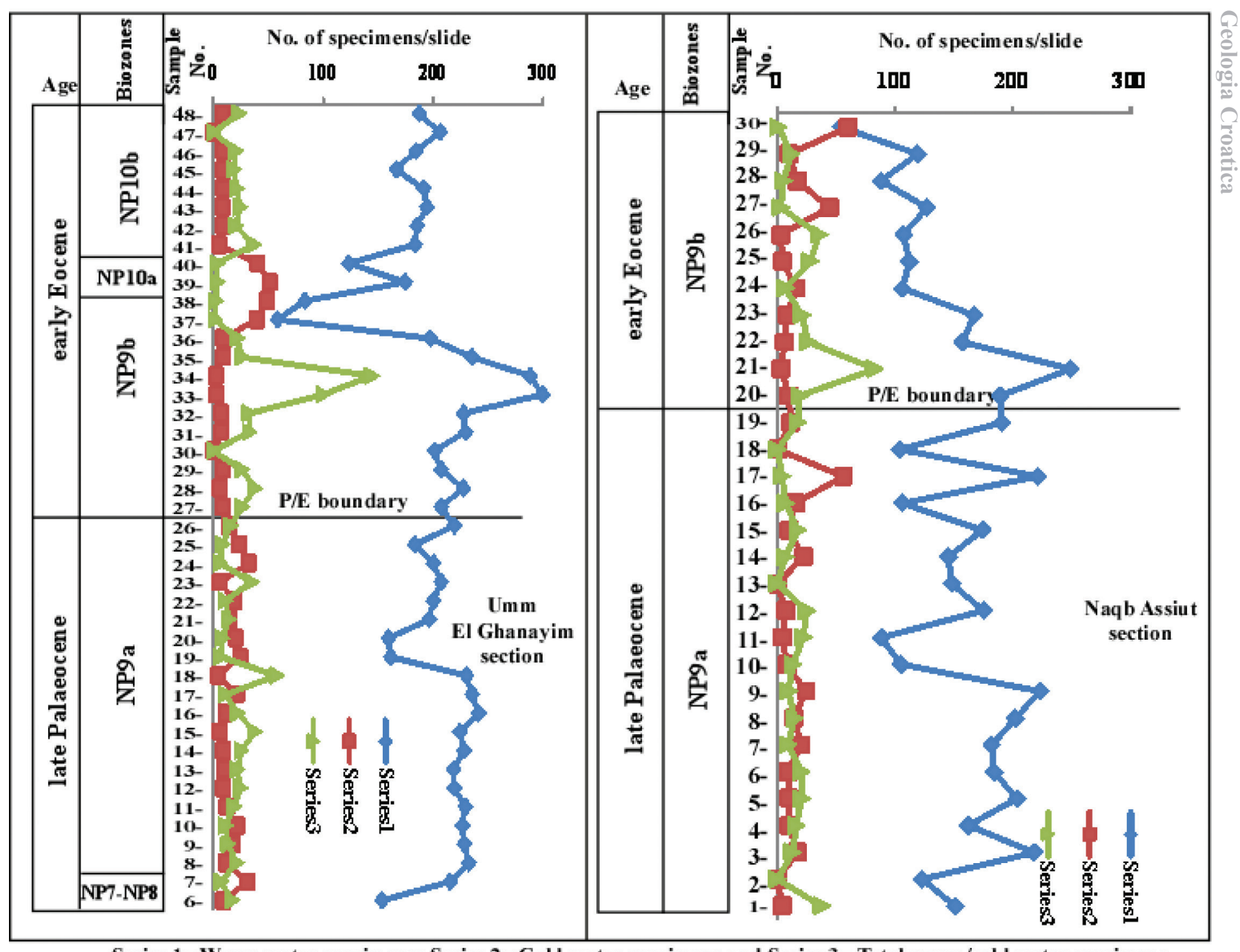

Series $1=$ Warm water specimens; Series $2=$ Cold water specimens and Series $3=$ Total warm/cold water specimens

Figure 7. Total warm and cold water specimens/slide and warm/cold water specimens in Umm El Ghanayim and Naqb Assiut sections.

graphic studies (MCINTYRE \& BE, 1967, HAQ \& LOHMANN, 1976, HAQ, 1980). In addition, C. pelagicus s. ampl. prevails in lower latitudes during mid-Palaeocene to early Eocene, where $C$. eopelagicus is documented in the early Eocene (HAQ \& LOHMANN, 1976) as in this study as characterizing global warming and early Eocene climatic optimum. Nevertheless, Coccolithus pelagicus dominates in the low latitudes throughout late Palaeoceneearly Eocene time, indicating nutrient improvement (JIANG \& WISE, 2006), which could also be the cause of our results too. Coccolithus pelagicus $\mathrm{s}$. ampl. dominates in assemblages together with Ericsonia subpertusa in our sections too. An increase of the relative abundance of Coccolithus pelagicus and Ercisonia subpertusa (C. subpertusus) during the PETM in the studied two sections was observed. They are interpreted as indicators of warm waters and were presumably adapted to oligotrophic environment based on their close association with the excursion taxa (discoasters, rhomboasters, tribrachiatus). It is evident that changes in the abundance of the calcareous nannofossil species can be interpreted as a response to palaeoecologic conditions such as palaeotemperature and nutrients. The acme of Ericsonia subpertusa (Coccolithus subpertusus) was recorded at the Dababyia section above the dissolution horizon with the LOs of D. anartios and Rhomboasters (base of NP9b) and coincides with the negative $\delta 13 \mathrm{C}$ value of the CIE (DUPUIS et al., 2003), which proves the aforementioned Benthic Foraminiferal Extinction Event (BFEE). These authors correlated this acme with that recorded at the Equatorial Pacific Ocean Drilling Site 865 (KELLY et al. 1996) and concluded that the acme of E. subpertusa is a global Acme possibly as a response to global warming. E. subpertusa (C. subpertusus) is common in the lower part of PETM, then reaches its acme in the middle part of the PETM in the Matulla section (West Central Sinai (ABU SHAMA et al., 2007) and coincides with the LO of Rhomboaster taxa. In the studied two sections, the relative abundance of $E$. subpertusa varies from frequent to common indicating warming conditions and eutrophication that occurred around the Palaeocene/Eocene boundary. The lower abundances of early Eocene oligotrophic, warm water Rhomboaster species are possible consequences of the aforementioned nutrient-richer water condition. This is also evident from the domination of eutrophic, warm water $C$. eopelagicus detected in the early Eocene at the Um El Ghanayim section. Today, large forms of C. pelagicus can be observed at lower latitudes and upwelling regions (ZIVERI et al., 2004, BAUMANN et al., 2000).

In the studied sections, semi-quantitative analyses of calcareous nannofossil assemblages were performed for the interval from NP7-8 to the basal part of the NP10 Zone. At the Um EL Ghanayim section, the number of warm water nannofossil taxa fluctuates between 6-17 species in the late Palaeocene (NP7-8 
Table 5. Calcareous nannofossils from Umm El Ghanayim section, grouped according to their paleoecological behaviour into warm water, cold water and non-characteristic species.

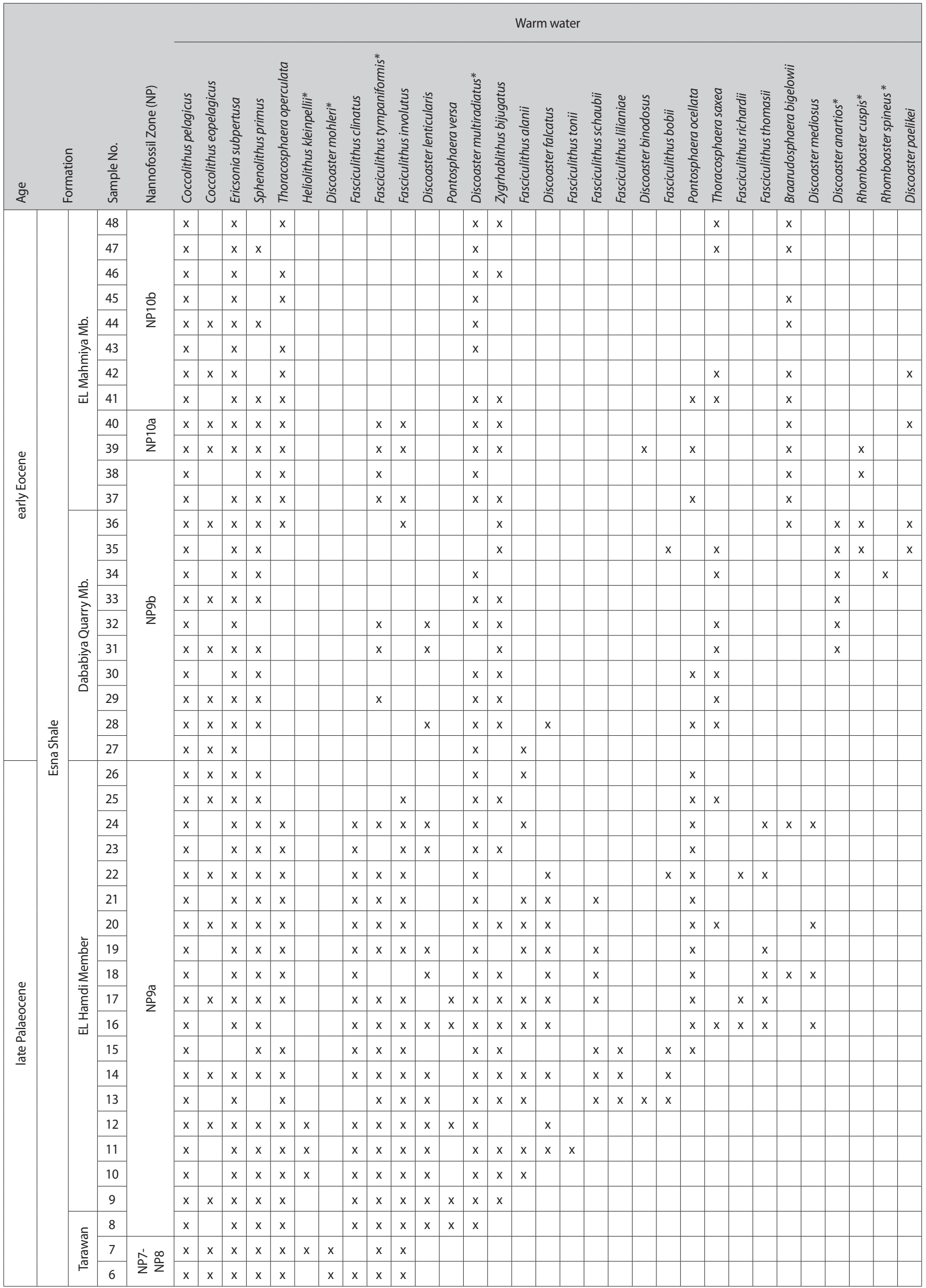




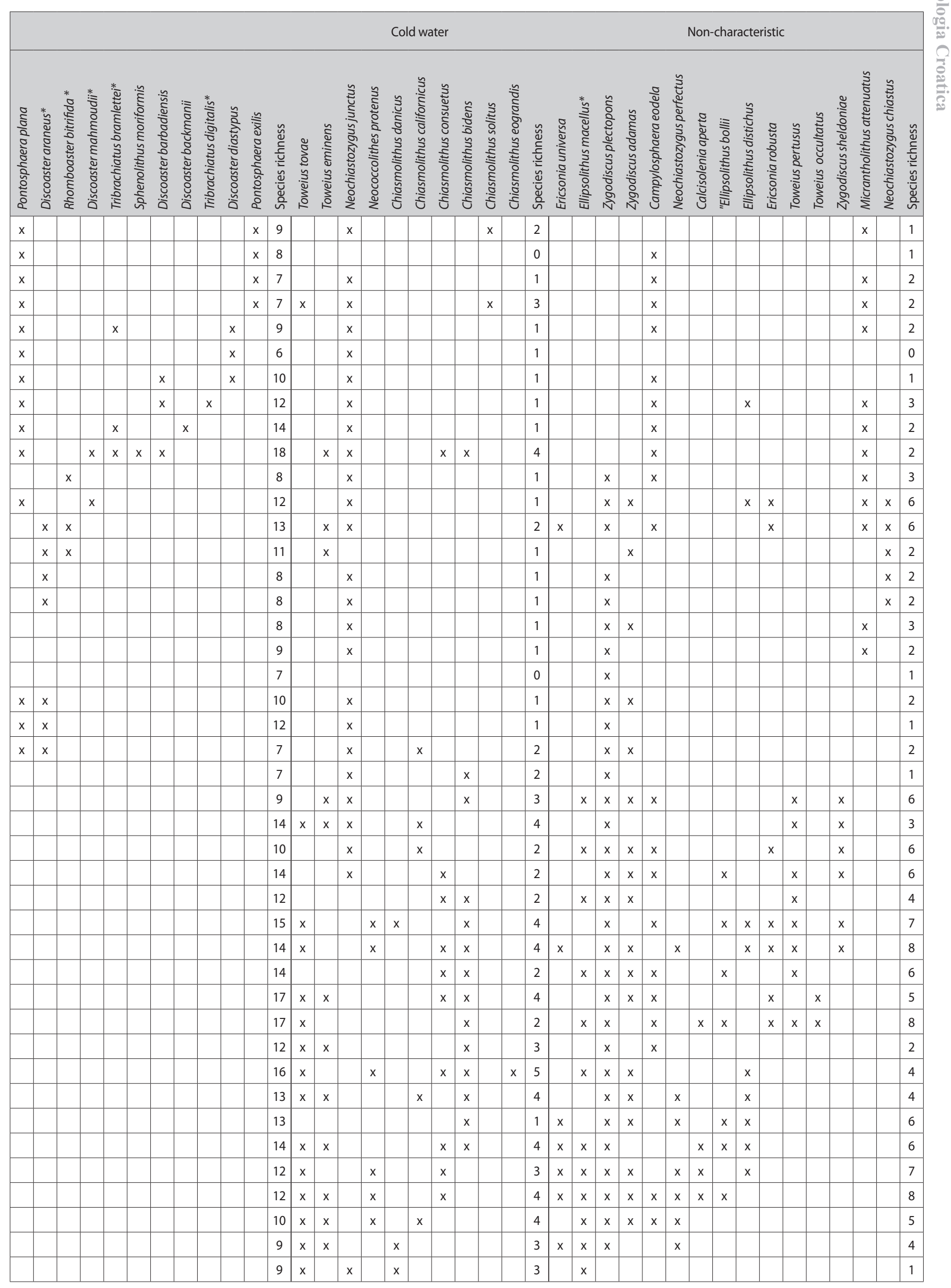


Table 6. Calcareous nannofossils from Naqb Assiut section, grouped according to their paleoecological behaviour into warm water, cold water and non-characteristic species

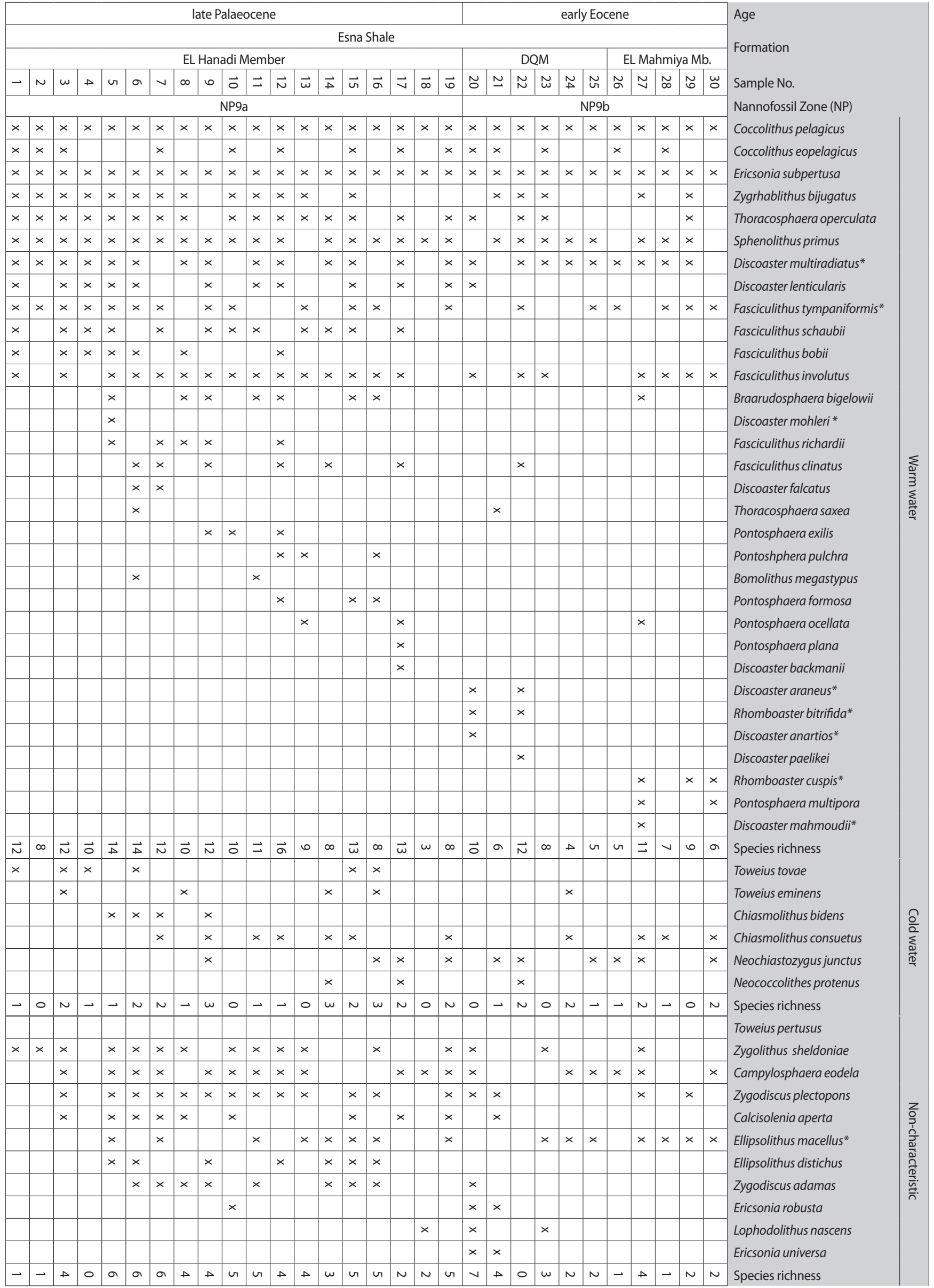


Zone to NP9a Subzone) and reaches a maximum in a single sample of 12 species in the NP9b of the early Eocene, while the cold water taxa gradually decreases around this boundary (Table 5). At the Naqb Assiut section, the warm water species range from 7 to 15 in number below the P/E boundary and decreases to 3 near this boundary. Nevertheless, warm-water Coccolithus eopelagicus, Discoaster multiradiatus, D. araneus, Ericsonia subpertrusa, Sphenolithus primus, Zygrhablithus bijugatus in conjunction to cold water Neochiastozygus junctus are the most common species of the late Palaeocene - early Eocene interval in the two studied sections. This suggests the Palaeocene-Eocene Thermal Maximum (PETM) with the pick in the samples 21 and 33 of the Naqb Assiut and Umm El Ghanayim sections respectively during this time interval (Figs. 6,7).

The warm, cold water and non-characteristic calcareous nannofossils near the P/E boundary at the Umm El Ghanayim and Naqb Assiut sections are shown in Tables 5 and 6. The vertical variations in the number of warm water and cold water species in these two sections are shown in Fig. 6. On the other hand, the total warm and cold water specimens/slide and warm/cold water specimens are shown in Fig. 7.

The last abundant appearance of Coccolithus subpertusus, and the frequent occurrence of excursion taxa including Discoaster araneus, D. anartios with rare Rhomboaster spp. across the PETM in four outcrops in Central Egypt are present in response to the beginning of the warming period (YOUSSEF, 2016).

\section{CONCLUSIONS}

Two upper Palaeocene - lower Eocene stratigraphic sequences at Kharga Oasis (the Umm El Ghanayim and Naqb Assiut sections) were studied biostratigraphically for their calcareous nannofossil content. The investigated interval comprises the upper part of the Tarawan and the Esna formations. The Esna Shale can be divided from the base to top as follows: the El Hanadi, the Dababiya Quarry and El Mahmiya members.

At the Umm El Ghanayim section, the Dababiya Quarry Member consists of three beds: an organic rich-clay layer $(15 \mathrm{~cm})$, brown-coloured fish debris and coprolite-rich laminated shale $(90$ $\mathrm{cm})$ and marls $(15 \mathrm{~cm})$. At the Naqb Assiut section, the Dababiya Quarry Member is composed of $20 \mathrm{~cm}$ of massive phosphatic marls rich with collophane grains at the base and $70 \mathrm{~cm}$ of marly limestone at the top. At the Naqb Assiut section, the P/E boundary interval is marked by the absence of the organic rich-clay layer (Bed no.1) of the Dababiya Quarry Member at the Dababiya section (GSSP) which may indicate the presence of a minor hiatus at the base of the Eocene. This minor unconformity could not be detected palaeontologically by means of nannofossils.

At the Gabal Umm El Ghanayim section, the exposed part of the Tarawan Chalk encompasses nannofossil zones NP6, NP7/8 and the basal part of the NP9a Subzone. The El Hanadi Member includes the middle and upper parts of the NP9a Subzone, and this means that a conformable relationship exists between these two units. In this section, the Dababiya Quarry Member includes the NP9b Subzone (early Eocene) and the El Mahmiya Member includes the upper NP9b, the NP10aand NP10b Subzones.

The base of the NP9b Subzone is delineated by the LO of Discoaster araneus at the G. Umm El Ghanayim section, while at the Gabal Naqb Assiut section, the first appearances of the nannofossil taxa Rhomboaster bitrifida, Discoaster araneus and D. anartios can be used to place the NP9a/NP9b subzonal boundary.
The current study indicates that the Umm El Ghanayim section appears to be stratigraphically complete, at least based on nannofossil biostratigraphic resolution, and spans a late Palaeocene - early Eocene time interval encompassing zones NP9 to the base of NP10. The Palaeocene-Eocene boundary is placed at the base of the Dababiya Quarry Member of the Esna Fomation.

Several nannofossil species became extinct in the upper part of the NP9a Subzone (late Palaeocene): Fasciculithus tonii, F. richardii, F. schaubii, F. thomasii, F. clinatus, F. lillianiae, Discoaster mediosus and D. mohleri. Other taxa which crossed the P/E boundary include: Campylosphaera eodela, Discoaster lenticularis, D. multiradiatus, Fasciculithus tympaniformis, F. involutus and Zygrhablithus bijugatus. The excursion taxa (Rhomboaster intermedia, R. bitrifida, R. cuspis, R. spineus, Discoaster araneus, D.anartios) in addition to D. paelikei and D. mahmoudii have their first occurrences within NP9b Subzone.

On the other hand, the species richness (diversity) fluctuates with a detected minimum around the Palaeocene/Eocene boundary and Fasciculithus spp. decreases in diversity and abundance above the Palaeocene/Eocene boundary in the studied sections which is in agreement with other aforementioned research in the region and globally.

\section{ACKNOWLEDGEMENT}

The authors wish to express their deep thanks to Ines GALOVIĆ and an anonymous reviewer for their insightful reviews, their suggestions and valuable comments on this manuscript.

\section{REFERENCES}

ABDEL-RAZIK, T.M. (1972): Comparative studies on the Upper Cretaceous-Early Palaeogene sediments on the Red Sea Coast, Nile Valley and Western Desert, Egypt.Proceedings of the $8^{\text {th }}$ Arab Petroleum Congress, 71(B-3), 1023, Algier.

ABU SHAMA, A.M., FARIS, M. \& Al-WOSABI, KH.A. (2007): Upper PalaeoceneLower Eocene calcareous nannofossil biostratigraphy and palaeoecology of Gebel Matulla section, south-western Sinai, Egypt Proc.- The $5^{\text {th }}$ International Conference of the Geology of Africa, Assiut, Egypt, 1, 33-51.

AGNINI, C., FORNACIARI, E., RAFFI, I., CATANZARITI, R., PALIKE, H., BACKMAN, I. \& RIO, D. (2014): Biozonation and biochronology of Palaeogene calcareous nannofossils from low and middle latitudes.- Newsletters on Stratigraphy, 47/2, 131-181.

AUBRY, M-P. (1992): Late Palaeogene nannoplankton evolution: a tale of climatic deterioration.- In: PROTHERO, D.R. \& BERGGREN, W.A. (eds.): Eocene-Oligocene Climatic and Biotic Evolution. Princeton Univ. Press, Princeton, NJ, 272-309.

AUBRY, M-P. (1996): Towards an Upper Palaeocene-Lower Eocene high resolution stratigraphy based on calcareous nannofossil stratigraphy.- Isr. J. Earth-Sci., 44, 239-253.

AUBRY, M-P. (1998): Late Palaeocene-Early Eocene climatic and biotic events in the marine and terrestrial records.- In: AUBRY, M-P., LUCAS, SG. \& BERGGREN, W.A. (eds.), Columbia Univ. Press, 158-203.

AUBRY, M-P. (1999): Late Palaeocene-early Eocene sedimentary history in western Cuba: implications for the LPTM and for regional tectonic history.- Micropaleontology, 45, 5-18.

AUBRY, M.-P., BERGGREN, W., CRAMER, B., DUPUIS, C., KENT, D., OUDA, KH., SCHMITZ, B. \& STEURBAUT, E. (1999): Palaeocene/Eocene boundary sections in Egypt.- In: OUDA, K.H. \& SOLIMAN, H. (eds.): Late Palaeocene-Early Eocene Events from Northern Africa to the Middle East. International Symposium in connection with First International Conference on the geology of Africa, Nov. 23-25.

AUBRY, M-P., OUDA, K., DUPUIS, C., BERGGREN, W.A., VAN COUVERING, J.A. $\&$ the Members of the Working Group on the Palaeocene/Eocene Boundary (2007): The Global Standard Stratotype-section and Point (GSSP) for the base of the Eocene Series in the Dababiya section (Egypt).- Episodes, 30/4, 271-286.

AUBRY, M.P. \& SALEM, R. (2013): The Dababiya Core: A window into Palaeocene to early Eocene depositional history in Egypt based on coccolith stratigraphy.Stratigraphy, 9/3-4, 287-346.

AWAD, G.H. \& GHOBRIAL, M.G. (1965): Zonal stratigraphy of the Kharga Oasis. Ministry of Industry, General Egyptian organization for geological research and Mining.- Geol. Surv., 34-77. 
AYYAD, S.N., FARIS, M., El NAHASS, H.A. \& SAAD, A.A. (2003): Planktonic Foraminiferal and calcareous nannofossil Biostratigraphy of the Upper CretaceousLower Eocene successions in the northeast Sinai, Egypt.- The $3^{\text {th }}$ International Conference, Assiut, Egypt, 1, 649-683.

BACKMAN, J. \& SHACKLETON, N.J. (1983): Quantitative biochronology of Pliocene and early Pleistocene calcareous nannofossils from the Atlantic, Indian and Pacific oceans.- Marine Micropaleontology, 8, 141-170. doi: 10.1016/03778398(83)90009-9

BAUMANN, K.-H., YOUNG, J.R., CACHAO, M. \& ZIVERI, P. (2000): Biometric study of Coccolithus pelagicus and its palaeoenvironmental utility, Bremen: 8. INA Conf. J. Nannoplankton Res., 22, p. 82.

BEADNELL, H.J.L. (1905): The topography and geology of the Fayum province of Egypt.- Survey Dept., Cairo, 101 p.

BOLLE, M-P., TANTAWY, A., PARDO, A., ADATTE, T., BURNS, S. \& KASSAB, A. (2000): Climatic and environmental changes documented in the Upper Palaeocene to Lower Eocene of Egypt.- Ecol. Geol. Helv., 93, 33-51.

BRALOWER, T.J. (2002): Evidence of surface water oligotrophy during the Palaeocene Eocene Thermal Maximum: nannofossil assemblage data from Ocean Drilling Program Site 690 Maud Rise, Weddell Sea.- Paleoceanograph., 7, 1-13.

BRAMLETTE, M.N. \& SULLIVAN, F.R. (1961): Coccolithophorids and related nannoplankton of the Early Tertiary in California.-- Micropaleontol., 7, 129-188. doi: $10.2307 / 1484276$

BUKRY, D. (1973): Low-latitude coccolith biostratigraphic zonation.- Proc. Deep Sea Drill. Proj., Initial Rep., 15, 685-703. doi: 10.2973/dsdp.proc.15.116.1973

DICKENS, G.R., O'NEIL, J.R., REA, D.K. \& OWEN, R.M. (1995): Dissociation of oceanic methane hydrate as a cause of the carbon isotope excursion at the end of the Palaeocene-- Paleoceanogr., 10, 965-971.

DUPUIS, C., AUBRY, M-P., STEURBART, E., BREGGREN, W.A., OUDA, K., MAGIONCALDA, R., CRAMER, B.S., KENT, D.V., SPEIJER, R.P. \& HEILMANNCLAUSEN, C. (2003): The Dababiya Quarry section: lithostratigraphy, geochemistry and paleontology.- Micropaleontol., 49/1, 41-59.

EDWARDS, A.R. (1968): The calcareous nannoplankton for Tertiary New Zealand climates, Tuatara, 16, 26-31.

EL-DAWY, H.M., OBAIDALLA, A.N., MAHFOUZ, H.K. \& ABDEL WAHED, S.A. (2016): Palaeocene-Eocene transition at Naqb Assiut, Kharga Oasis, Western Desert, Egypt: Stratigraphical and paleoenvironmental inferences.- Jour. of Afr. Earth Sci., 117, 207-222.

FARIS, M. (1988a): Late Cretaceous-Early Tertiary calcareous nannofossils from ElQusaima area, NE Sinai, Egypt.- Bull. Fac. Science Qena (Egypt), 2/1, 263-275.

FARIS, M. (1988b): Remarks on Late Palaeocene-Early Eocene calcareous nannofossils from Gebel Um Huetat, Safaga area, Egypt.- Bull. Fac. Science Qena (Egypt), 2/1, 277-297.

FARIS, M., ALLAM, A. \& MARZOUK, A.M. (1989): Biostratigraphy of the Late Cretaceous - Early Tertiary rocks in the Nile Valley (Qena region), Egypt.-Geol. Surv. Egypt, 15, 287-300.

FARIS, M. \& STROUGO, A. (1998): The Lower Libyan in Farafra (Western Desert) and Luxor (Nile Valley): Correlation by calcareous nannofossils.- M.E.R.C., Ain Shams University Earth Science Series, 12, 137-156.

FARIS, M., ABD El-HAMEED, A.T., MARZOUK, A.M. \& GHANDOUR, I.M. (1999): Early Palaeogene calcareous nannofossil and planktonic foraminiferal biostratigraphy in Central Egypt.- Neues Jahrbuch für Geology und Palaöntology, 213/2, 261-288.

FARIS, M., El-DEEB, W. \& MANDOUR, M. (2000): Biostratigraphy of some Upper Cretaceous/Lower Eocene successions in North and Southwest Sinai, Egypt.-Ann. Geol. Surv. Egypt, 23, 135-161.

FARIS, M. \& ZAHRAN, E. (2002): Calcareous nannofossil biostratigraphy of the Late Palaeocene/Early Eocene of El-Bruk area, North Central Sinai, Egypt.- Egyptian Journal of Paleontology, 2, 359-369.

FARIS, M., AYYAD, S.N., El NAHASS, H.A. \& Al WOSABI, KH.A.A. (2005): Integrated Planktonic and Calcareous Nannofossil Biostratigraphy of the Upper Cretaceous-Lower Eocene Formations, West Central Sinai, Egypt.- The $5^{\text {th }}$ International Conference on the Geology of Africa, 2, 769-791.

FARIS, M. \& ABU SHAMA, A.M. (2007): Nannofossil biostratigraphy of the Palaeocene-Eocene succession in the Thamad area, East Central Sinai, Egypt.- Micropaleontogy, 53/1-2,127-144.

FARIS, M. \& SALEM, R.F. (2007): Palaeocene-early Eocene calcareous nannofossil biostratigraphy in west central Sinai, Egypt.- Proceeding of the $8^{\text {th }}$ Conference Geology of Sinai for Development, Ismailia, 1-14.

FARIS, M. \& FAROUK, S. (2015): Calcareous nannofossils of the Palaeocene-Eocene transition in four sections from Egypt.- Turk. J. Earth Sci., 24, 585-606.

FARIS, M., GHANDOUR, I.M., ZAHRAN, E. \& MOSA, G. (2015): Calcareous nannoplankton changes during the Palaeocene-Eocene Thermal Maximum in West Central Sinai, Egypt.- Turk. Jour. of Earth Sci., 24, 475-493.

GIBBS, S., BRALOWER, T.J., BOWN, P.R., ZACHOS, J.C. \& BYBELL, L.M. (2006a): Shelf and openocean calcareous phytoplankton assemblages across the Palaeocene-
Eocene Thermal Maximum: Implications for global productivity gradients.- Geology, 34, 233-236.

GIBBS, S.J., BOWN, P.R., SESSA, J.A., BRALOWER, T.J. \& WILSON, P.A. (2006b): Nannoplankton extinction and origination across the Palaeocene-Eocene Thermal Maximum.- Science, 314, 1770-1773.

GHANDOUR, I.M., ABD EL-HAMEED, A.T., FARIS M., MARZOUK, A \& MAEJIMA, W. (2004): Textural, Mineralogical and Microfacies Characteristics of the Lower Palaeogene Succession at the Nile Valley and Kharga Oasis Regions, Central Egypt.- Jour. Geosci., Osaka Univ., 47/4, 39-53.

HAQ, B.U. (1980): Biogeographic history of Miocene calcareous nannoplankton and paleoceanography of the Atlantic ocean.- Micropaleontology, 26/4, 414-43. doi: $10.2307 / 1485353$

HAQ, B.U. \& LOHMANN, G.P. (1976): Early Cenozoic calcareous nannoplankton biogeography of the Atlantic Ocean. Marine Micropaleontology, 1, 119-194. doi: 10.1016/0377-8398(76)90008-6

HAY, W.W. (1964): The use of the electron microscope in the study of fossils. Smithsonian Institution.

HAY, W.W., MOHLER, H.P., ROTH, P.H., SCHMIDT, R.R. \& BORDEAUZ, J.E. (1967): Calcareous nannoplankton zonation of the Cenozoic of the Gulf Coast and Caribbean-Antillean area, Transoceanic Correlation.- Transactions of the Gulf Coast Association of Geological Societies, 17, 428-480.

HERMINA, M. (1990): The surroundings of Kharga, Dakhla and Farafra Oases.- In: SAID, R. (ed.): The Geology of Egypt, Rotterdam, Balkema, 734 p.

HEWAIDY, A.A. \& FARIS, M. (1989): Biostratigraphy and palaeoecology of the Late Cretaceous-Early Tertiary sequence in Wasif area, Safaga District, Eastern Desert, Egypt.- Delta Jour. of Sci., 13/4,1953-1975.

JIANG, WISE, S.W. (2006):Surface-water chemistry and fertility variations in the tropical Atlantic across the Palaeocene/Eocene thermal maximum as evidenced by calcareous nannoplankton from ODP Leg 207, Hole 1259B.- Revesta Micropaleontology, 49/4, 227-244.

KAHN, A. \& AUBRY, M.-P. (2004): Provincialism associated with the Palaeocene/ Eocene Thermal Maximum: temporal constraint.- Marine Micropaleontology, 52, $117-131$.

KATZ, M.E., PAK, D.K., DICKENS, G.R. \& MILLER, K.G. (1999): The source and fate of massive carbon input during the latest Palaeocene thermal maximum.- Science, 286/5444, 1531-1533.

KELLY, D.C., BRALOWER, T.J., ZACHOS, J.C., PREMOLI SILVA, I. \& THOMAS, E. (1996): Rapid diversification of planktonic foraminifera in the tropical Pacific (ODP Site 865) during the late Palaeocene thermal maximum.- Geology, 24, 423-426.

KENNETT, J.P. \& STOTT, L.D. (1991): Abrupt deep-sea warming, palaeoceanographic changes and benthic extinctions at the end of the Palaeocene.- Nature, 353, 225-229. doi: $10.1038 / 353225 \mathrm{a} 0$

KHALIFA, H. (1970): Geology of Gabal E1 Shaghab area, near Esna.- MSc. Memoir, Department of Geology, University of Assiut, U.A.R., 85 p., 29 figs, 1 map.

KHALIL, H. \& Al SAWY, S. (2014): Integrated biostratigraphy, stage boundaries and Paleoclimatology of the Upper Cretaceous-Lower Eocene successions in Kharga and Dakhala Oases, Western Desert, Egypt.- Jour. of Afr. Earth Sci., 96, 220-242.

KHOZYEM, H., ADATTE, T., SPANGENBERG, J.E., TANTAWY, A.A. \& KELLER, G. (2013): Palaeoenvironmental and climatic changes during the PalaeoceneEocene Thermal Maximum (PETM) at the Wadi Nukhul Section, Sinai, Egypt.Journal of the Geological Society, London, 170, 341-352.

KHOZYEM, H., ADATTE, T., KELLER, G., TANTAWY, A.A. \& SPANGENBERG, J.E. (2014): The Palaeocene-Eocene GSSP at Dababiya, Egypt-Revisited.- Episodes, $37 / 2,78-85$.

LUNING, S., MARZOUK, A. \& KUSS, J. (1998): The Palaeocene of central east Sinai, Egypt: 'sequence stratigraphy' in monotonous hemipelagites.- Journal of Foram. Res, 28, 19-39.

MARTINI, E. (1971): Standard tertiary and quaternary calcareous nannoplankton zonation.- In: FARINACCI, A. (ed.): Proceedings of the 2nd Planktonic Conference, Roma.- Tecnoscienza, Rome, 739-785.

MCINTYRE, A. \& BE, A.W.H., (1967): Modern coccolithophoridae of the Atlantic Ocean-I.Placoliths and cyrtoliths.- Deep-Sea Res. Part A 14, 561-597.

MUTTERLOSE, J., LINNERT, C. \& NORRIS, R. (2007): Calcareous nannofossils from the Palaeocene-Eocene Thermal Maximum of the equatorial Atlantic (ODP Site 1260B): Evidence for tropical warming.- Mar. Micropaleontol., 65,13-31.

OKADA, K.H. \& BUKRY, D. (1980): Supplementary modifications and introduction of code numbers to the low latitude coccolith biostratigraphic zonation.- Mar. Micropaleontol., 5, 321-325.

OUDA, K.H., BERGGREN, W.A. \& ABDEL SABOUR, A. (2016a): Biostratigraphy of the Upper Palaeocene-Lower Eocene succession of Gebel El Aguz, northeastern Kharga Oasis, Western Desert, Egypt.- Revue de Paléobiologie, Genève, 3/1, 341-371.

OUDA, K.H., BERGGREN, W.A. \& ABDEL SABOUR, A. (2016b): Upper PalaeoceneLower Eocene biostratigraphy of Darb Gaga, Southeastern Kharga Oasis Western Desert, Egypt.- Journal of Afr. Earth Sci., 118, 12-23. 
PERCH-NIELSEN, K. (1985): Mesozoic calcareous nannofossils.- In: BOLLI, H.M., SAUNDERS, J.B. \& PERCH-NIELSEN, K. (eds.): Plankton Stratigraphy. Cambridge Univ. Press, 329-426.

ROMEIN, A.J.T. (1979): Lineages in early Palaeogene calcareous nannoplankton.Utrecht Micropalaeontological Bulletins, 22, 1-230.

SAID, R. (1990): Cenozoic. The geology of Egypt, Puplished for the Egyptian General Petroleum Coroporation, Conoco, Hurghada, Inc., and Repsol Exploracion, 1, 451-486.

SPEIJER, R.P., SCHMITZ, B. \& LUGER, P. (2000): Stratigraphy of late Palaeocene events in the Middle East: Implications for low to middle latitude successions and correlations.- Journal of Geological Society, London, 157, 37-47.

STROUGO, A. \& FARIS, M. (1993): Palaeocene/Eocene Stratigraphy of Wadi El Dakhl, Southern Galala Pl.au. M.E.R.C.-Ain Shams University Earth Science Series, 7, 49-62.

TANTAWY, A.A. (1998): Stratigraphical and paleontological studies on some Palaeocene-Eocene succession in Egypt.- Ph.D. Thesis, Assiut University, 273 p.

TANTAWY, A.A. (2006): Calcareous nannofossils of the Palaeocene-Eocene transition at Qena Region, Central Nile Valley, Egypt.- Micropaleontology, 52, 193-222.

THOMAS, E. (1998): Biogeography of the late Palaeocene benthic foraminiferal extinction.- In: AUBRY, M.-P., LUCAS, S.G. \& BERGGREN, W.A. (eds.): Late Palaeocene-Early Eocene: Climatic and Biotic Events in the Marine and Terrestrial Records. Columbia Univ. Press, New York, NY, 214-235.

THOMAS, E. \& SHACKLETON, N.J. (1996): The latest Palaeocene benthic foraminiferal extinction and stable isotope anomalies.- In: KNOX, R.O., CORFIELD, R.M.

\section{LIST OF CALCAREOUS NANNOFOSSIL TAXA}

Bomolithus megastypus (BRAMLETTE \& SULLIVAN, 1961) BOWN, 2005

Braarudosphaera bigelowii (GRAN \& BRAARUD, 1935) DEFLANDRE, 1947

Calciosolenia aperta (HAY \& MOHLER, 1967) BOWN, 2005

Campylosphaera eodela BUKRY \& PERCIVAL, 1971

Chiasmolithus bidens (BRAMLETTE \& SULLIVAN, 1961) HAY \& MOHLER, 1967

Chiasmolithus californicus (SULLIVAN, 1964) HAY \& MOHLER, 1967

Chiasmolithus consuetus (BRAMLETTE \& SULLIVAN, 1961) HAY \& MOHLER, 1967

Chiasmolithus danicus (BROTZEN, 1959) HAY \& MOHLER, 1967

Chiasmolithus eograndis PERCH-NIELSEN, 1971

Chiasmolithus solitus (BRAMLETTE \& SULLIVAN, 1961) LOCKER, 1968

Coccolithus eopelagicus (Bramlette \& Riedel, 1954) Bramlette \& Sullivan 1961

Coccolithus pelagicus (WALLICH, 1877) SCHILLER, 1930

Discoaster anartios BYBELL \& SELF-TRAIL, 1995

Discoaster araneus BUKRY, 1971

Discoaster backmanii AGNINI \& FORNACIARI, 2008

Discoaster barbadiensis TAN, 1927

Discoaster binodosus MARTINI, 1958

Discoaster diastypus BRAMLETTE \& SULLIVAN, 1961

Discoaster falcatus BRAMLETTE \& SULLIVAN, 1961

Discoaster lenticularis BRAMLETTE \& SULLIVAN, 1961

Discoaster mahmoudii PERCH-NIELSEN, 1981

Discoaster mediosus BRAMLETTE \& SULLIVAN, 1961

Discoaster mohleri BRAMLETTE \& PERCIVAL, 1971

Discoaster multiradiatus BRAMLETTE \& REIDEL, 1954

Discoaster paelikei AGNINI et al., 2008

Ellipsolithus bollii PERCH-NIELSEN, 1977

Ellipsolithus distichus (BRAMLETTE \& SULLIVAN, 1961) SULLIVAN, 1964
\& DUNAY, R.E. (eds.): Correlation of the Early Palaeogene in Northwest Europe. Geological Society of London Special Publication, 101, 401-441.

TREMOLADA, F. \& BRALOWER, T.J. (2004): Nannofossil assemblage fluctuations during the Palaeocene-Eocene Thermal Maximum at Sites 213 (Indian Ocean) and 401 (North Atlantic Ocean): palaeoceanographic implications.- Marine Micropaleontol., 52, 107-116.

VON SALIS, K., OUDA, K., SAAD EI DIN, M., TANTAWY, A.A. \& BEMASCONI, S. (1998): Calcareous nannofossil, foraminifera and stable isotope studies from the $\mathrm{P} / \mathrm{E}$ boundary section in Egypt.- Strata, Actes du laboratoire de Geologie Sedimentaire et Paleontologie de l'Université Paul-Sabatier, 1, 113-115.

YOUSSEF, M. (2009): High resolution calcareous nannofossil biostratigraphy and palaeoecology across the Latest Danian Event (LDE) in central Eastern Desert, Egypt.- Marine Micropaleontology, 72, 111-128.

YOUSSEF, M. (2015): Evaluation of foraminifera and calcareous nannofossils changes across the Late Palaeocene/Early Eocene transition at Nag El-Quda section, Upper Nile Valley, Egypt.- Geosciences Jour., 19/4, 641-654.

YOUSSEF, M. (2016): Calcareous nannofossils and paleoenvironments of the Palaeocene-Eocene thermal maximum (PETM) interval in central Egypt.- Jour. of Afr. Earth Sci., 114, 203-219.

ZIVERI, P., BAUMANN, K.H., BÖCKEL, B., BOLLMANN, J. YOUNG, J.R. (2004): Biogeography of selected Holocene coccoliths in the Atlantic Ocean.- In: THIERSTEIN, H.R. \& YOUNG, J.R. (eds.): Coccolithophores from Molecular Processes to Global Impact. Springer, Berlin, 403-453.doi: 10.1007/978-3-66206278-4 15

Ellipsolithus macellus (BRAMLETTE \& SULLIVAN, 1961) SULLIVAN, 1964

Ericsonia robusta (BRAMLETTE \& SULLIVAN, 1961) EDWARDS \& PERCH-NIELSEN, 1975

Ericsonia subpertusa HAY \& MOHLER, 1967

Ericsonia universa (WIND \& WISE, 1977) ROMEIN, 1979

Fasciculithus alanii PERCH-NIELSEN, 1971

Fasciculithus clinatus BUKRY, 1971

Fasciculithus bobii PERCH-NIELSEN, 1971

Fasciculithus involutus BRAMLETTE \& SULLIVAN, 1961

Fasciculithus lillianiae PERCH-NIELSEN, 1971

Fasciculithus richardii PERCH-NIELSEN, 1971

Fasciculithus thomasii PERCH-NIELSEN, 1971

Fasciculithus tonii PERCH-NIELSEN, 1971

Fasciculithus schaubii HAY \& MOHLER, 1967

Fasciculithus tympaniformis HAY \& MOHLER in HAY et al., 1967

Heliolithus kleinpelli SULLIVAN, 1964

Lophodolithus nascens BRAMLETTE \& SULLIVAN, 1961

Micrantholithus attenuatus BRAMLETTE \& SULLIVAN, 1961

Neochiastozygus chiastus (BRAMLETTE \& SULLIVAN, 1961) PERCH-NIELSEN, 1971

Neochiastozygus junctus (BRAMLETTE \& SULLIVAN, 1961) PERCH-NIELSEN, 1971

Neochiastozygus perfectus PERCH-NIELSEN, 1971

Neococcolithes protenus (BRAMLETTE \& SULLIVAN, 1961) BLACK, 1967

Pontosphaera exilis (BRAMLETTE \& SULLIVAN, 1961) ROMEIN, 1979

Pontosphaera formosa (BUKRY \& BRAMLETTE, 1969) ROMEIN, 1979

Pontosphaera multipora (KAMPTNER, 1948 ex DEFLANDRE in DEFLANDRE \& FERT, 1954) ROTH, 1970

Pontosphaera ocellata (BRAMLETTE \& SULLIVAN, 1961) PERCH-NIELSEN, 1984

Pontosphaera plana (BRAMLETTE \& SULLIVAN, 1961) HAQ, 1971 
Pontosphaera pulchra (DEFLANDRE in DEFLANDRE \& FERT, 1954) ROMEIN, 1979

Pontosphaera versa (BRAMLETTE \& SULLIVAN, 1961) SHERWOOD, 1974

Rhomboaster bitrifida ROMEIN, 1979

Rhomboaster cuspis BRAMLETTE \& SULLIVAN, 1961

Sphenolithus moriformis (BRONNIMANN \& STRADNER, 1960) BRAMLETTE \& WILCOXON 1967

Sphenolithus primus PERCH-NIELSEN 1971

Thoracosphaera operculata BRAMLETTE \& MARTINI, 1964

Thoracosphaera saxea STRADNER, 1961

Toweius eminens (BRAMLETTE \& SULLIVAN, 1961) PERCHNIELSEN, 1971

Toweius occultatus (LOCKER, 1967) PERCH-NIELSEN, 1971
Toweius pertusus (SULLIVAN, 1965) ROMEIN, 1979

Toweius tovae PERCH-NIELSEN, 1971

Tribrachiatus bramlettei (BRONNIMANN \& STRADNER, 1960) PROTO DECIMA et al., 1975

Tribrachiatus digitalis AUBRY, 1996

Rhomboaster cuspis BRAMLETTE \& SULLIVAN, 1961

Rhomboaster spineus (SHANK \& STRADNER, 1971) PERCHNIELSEN, 1984

Zygodiscus adamas BRAMLETTE \& SULLIVAN, 1961

Zygodiscus sheldoniae BOWN, 2005

Zygodiscus plectopons BRONNIMANN \& STRADNER, 1961 Zygrhablithus bijugatus (DEFLANDRE in DEFLANDRE \& FERT, 1954) DEFLANDRE, 1959

Plate 1

1) Braarudosphaera bigelowii (GRAN \& BRAARUD, 1935) DEFLANDRE, 1947, sample 12, Naqb Assiut section.

2) Chiasmolithus bidens (BRAMLETTE \& SULLIVAN, 1961) HAY \& MOHLER 1967, sample 6, Um El Ghanayim section.

3, 4) Campylosphaera eodela BUKRY \& PERCIVAL, 1971, sample 8, Um El Ghanayim section

5, 6) Ericsonia subpertusa HAY \& MOHLER, 1967, sample 8, Naqb Assiut section.

7) Ellipsolithus distichus(BRAMLETTE \& SULLIVAN, 1961) SULLIVAN, 1964, sample 20, Um El Ghanayim section.

8) Ellipsolithus macellus (BRAMLETTE \& SULLIVAN, 1961) SULLIVAN, 1964, sample 23, Naqb Assiut section.

9, 10) Fasciculithus clinatus BUKRY 1971, sample 5, Naqb Assiut section.

11, 14) Fasciculithus involutus BRAMLETTE \& SULLIVAN, 1961.

11, 12) Sample 14, Naqb Assiut section.

13, 14) Sample 20, Naqb Assiut section.

15) Fasciculithus tympaniformis HAY \& MOHLER in HAY et al. 1967, sample 12, Um El Ghanayim section.

16) Heliolithus kleinpellii SULLIVAN (1964) sample 5, Um El Ghanayim section.

17, 18) Micrantholithus attenuatus BRAMLETTE \& SULLIVAN (1961) sample 44, Um El Ghanayim section.

19 , 20) Neochiastozygus junctus (BRAMLETTE \& SULLIVAN, 1961) sample 23, Um El Ghanayim section.

21-23) Zygrhablithus bijugatus (DEFLANDRE in DEFLANDRE \& FERT, 1954) DEFLANDRE, 1959.

21, 22) Sample 13 Naqb Assiut section.

23) Sample 41, Um El Ghanayim section.

24, 25) Calciosolenia aperta (HAY \& MOHLER, 1967), BOWN, 2005, sample 9, Um El Ghanayim section.

$\mathbf{2 6}$, 27) Zygodiscus plectopons BRAMLETTE \& SULLIVAN, 1961, sample 7, Naqb Assiut section. 

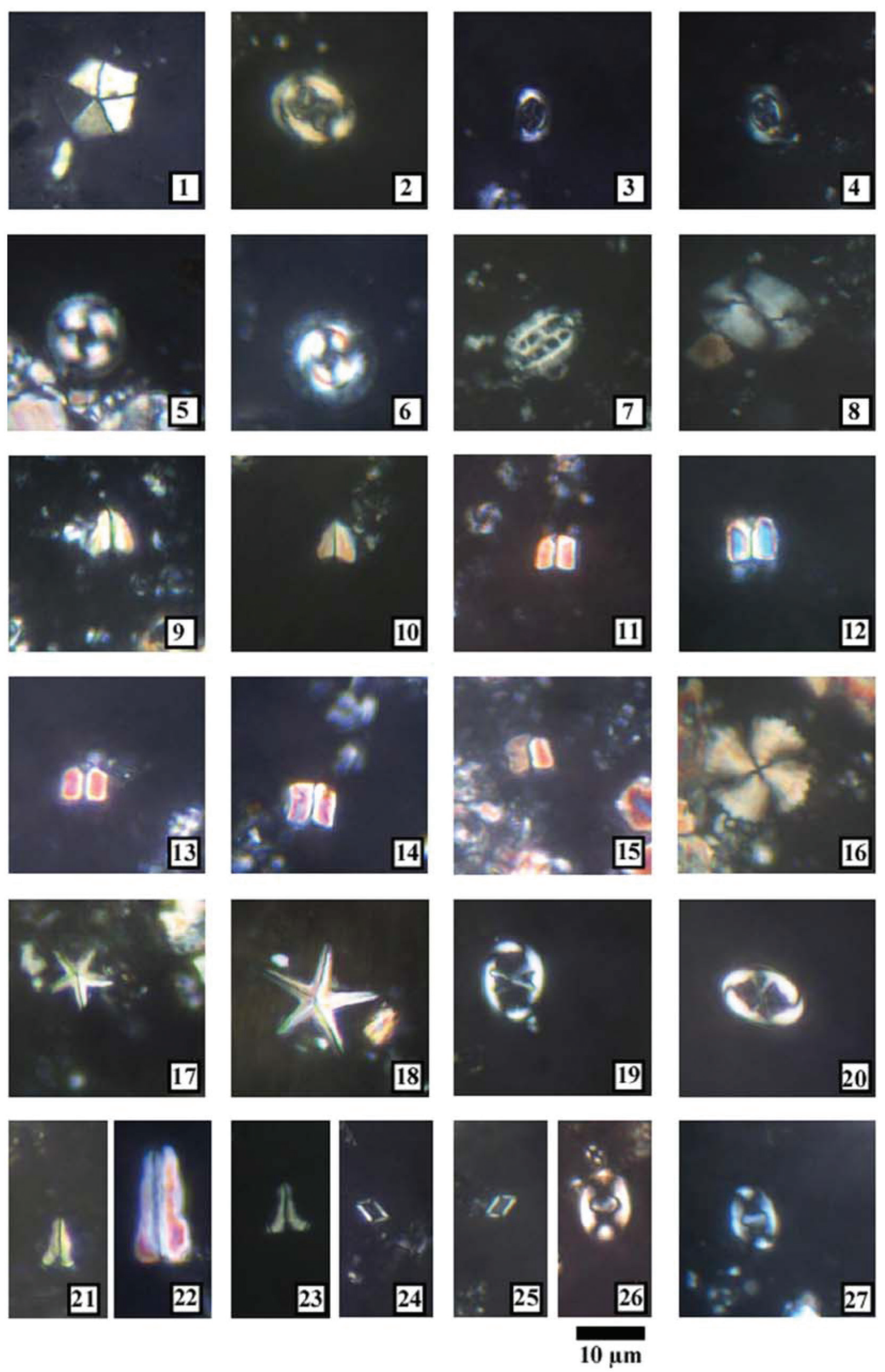
Plate 2

1-4) Discoaster araneus BUKRY, 1971, 1, 2) sample 20, Naqb Assiut section

3, 4) Sample 35, Um El Ghanayim section.

5) Discoaster anartios BYBELL \&SELF-TRAIL 1995, sample 20, Naqb Assiut section.

6) Discoaster barbadiensis TAN 1927, sample 42, Um El Ghanayim section.

7) Discoaster diastypus BRAMLETTE \& SULLIVAN 1961, sample 42, Um El Ghanayim section.

8) Discoaster falcatus BRAMLETTE \& SULLIVAN 1961, sample 22, Um El Ghanayim section.

9) Discoaster mahmoudii PERCH-NIESEN 1981, sample 27, Naqb Assiut section.

10) Discoaster multiradiatus BRAMLETTE \& REIDEL 1954, sample 5, Naqb Assiut section.

11 , 12) Discoaster paelikei AGNINI et al. 2008, sample 36, Um El Ghanayim section.

13, 14) Pontosphaeraexilis (BRAMLETTE \& SULLIVAN, 1961) ROMEIN, 1979, sample 9, Naqb Assiut section.

15) Pontosphaera plana (BRAMLETTE \& SULLIVAN, 1961) HAQ 1971, sample 17, NaqbAssiut section

16) Coccolithus eopelagicus (WALLICH, 1877) SCHILLER, 1930, sample 44, Umm El Ghanayim section

17) Rhomboaster bitrifida ROMEIN 1979, sample 38, Um El Ghanayim section.

18) Rhomboaster cuspis BRAMLETTE \& SULLIVAN 1961, sample 27, Naqb Assiut section

19) Sphenolithus moriformis (BRONNIMANN \& STRADNER, 1960) BRAMLETTE \& WILCOXON, 1967, sample 39, Um El Ghanayim section.

20) Sphenolithus primus PERCH-NIELSEN 1971, sample 19, Um El Ghanayim section.

21) Tribrachiatus bramlettei (BRONNIMANN \& STRADNER, 1960) PROTODECIMA et al., 1975, sample 39, Um El Ghanayim section.

22) Tribrachiatus digitalis AUBRY 1996, sample 41, Um El Ghanayim section.

23) Toweius eminens (BRAMLETTE \& SULLIVAN, 1961), PERCH NIELSEN, 1971, sample 16, Naqb Assiut section.

24) Thoracosphaera operculata BRAMLETTE \& MARTINI (1964), sample 4, Um El Ghanayim section. 

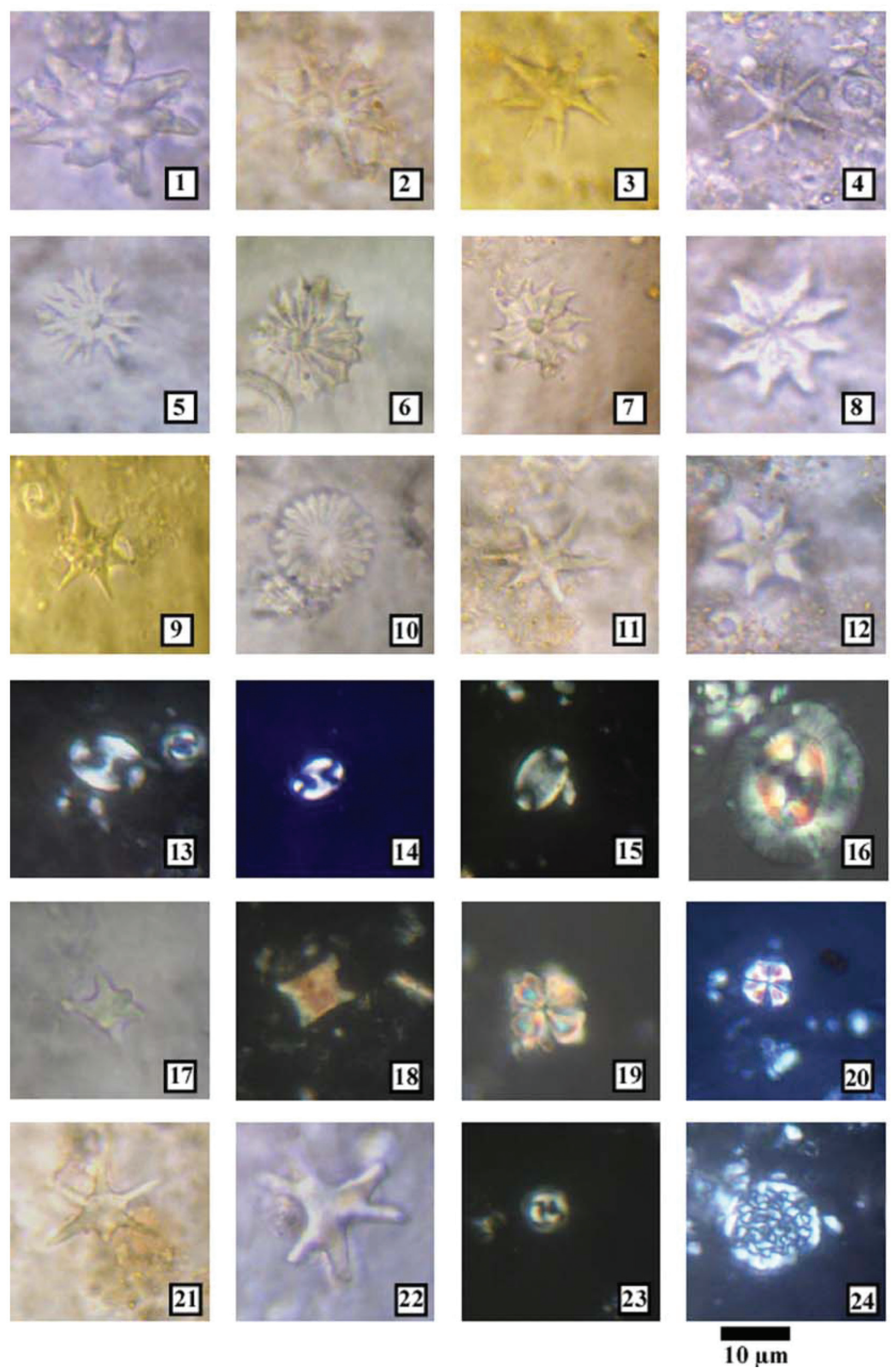
